\title{
Toughening and stiffening of starch food extrudates through the addition of cellulose fibres and minerals
}

\author{
C. G. Skamniotis ${ }^{\mathrm{a}}$, Y. Patel ${ }^{\mathrm{a}}$, M. Elliott ${ }^{\mathrm{b}}$, M. N. Charalambides ${ }^{\mathrm{a}}$ \\ ${ }^{a}$ Department of Mechanical Engineering, Imperial College London, London SW7 2AZ, United Kingdom \\ b Mars Petcare, Oakwell Way, Birstall, Batley, WF17 9LU, UK \\ E-mail: c.skamniotis13@imperial.ac.uk; m.charalambides@imperial.ac.uk
}

\begin{abstract}
Pet food, one of the largest type of commercial packaged foods, continuously sets new challenges, amongst them the possibility to enhance palatability via adjusting product composition. This will optimise texture perception across consumer groups of diverse chewing capabilities, as well as improve food oral breakdown efficiency with further impact on metabolic health and nutrient bioavailability in the digestive process. Our aim is to pioneer new methods of controlling texture by answering longstanding questions such as the impact of nutrients on the mechanical properties of foods. The impact of cellulose fibres and minerals on the fracture toughness and stiffness properties of starch food extrudates is investigated for the first time through employing tensile tests and two fracture toughness tests namely Essential Work of Fracture (EWF) and cutting, on four different compositions. Fibres alone are found to increase stiffness (stiffening) and toughness (toughening) whereas minerals decrease stiffness (softening) with a minor influence on toughness. Interestingly, fibres and minerals combined maximise toughening at $28 \%$ compared to pure starch, due to the synergistic effect of fibre-matrix debonding and fibre breakage mechanisms at the crack tip. These new results indicate that texture can be significantly altered through the addition of minerals and short fibres. Such information is critical in the design of products that need to satisfy both nutritional and textural criteria.
\end{abstract}

Keywords: starch food extrudates, cellulose fibres, minerals, toughening mechanisms, essential work of fracture, orthogonal cutting

\section{Introduction}

Like all carnivores, dogs display sharp, pointed teeth, and have short gastrointestinal tracts designed for meat consumption (Ockerman and Hansen 1999). However, over thousands of years, dogs have managed to adapt and survive on meat as well as non-meat food waste and now can be fed a variety of foods (Ockerman and Hansen 1999, Axelsson, Ratnakumar et al. 2013). The pet food industry has been continuously growing over the past four decades, producing products for domesticated animals that are formulated according to their nutritional needs (Arenofsky 2018). These typically consist of meat, meat by-products, cereals, grain, vitamins, and minerals (Hand, Hefferren et al. 1995). As long ago as in 1974 in the United States (US) about 300 manufacturers were producing more than seven million tons of pet food per year, already indicating one of the largest categories of any packaged food (Nutrition 1974). Owners could choose from more than 3000 products, including dry, canned, semi-moist types as well as snacks such as biscuits, kibbles and treats (Nutrition 1974). These figures have more than tripled in the past decade as pet parenting 
has become global with worldwide pet products and services reaching a record 100 USD billion in 2014 and total expenditure growing about 50\% faster than the retail sector (Arenofsky 2018).

Commercial products are assessed against quality criteria, amongst which nutritional completeness and balance, digestibility and safety are the most important (Zicker 2008). Recently, palatability is also receiving remarkable attention as not only it dictates whether food texture is appealing enough to be consumed and thus the commercial success of a product (Nahm Jr 1982, Axelrod 1993, Bierer 2007) but also it links to food breakdown efficiency (Çakır, Vinyard et al. 2012), oral care (Hand, Hefferren et al. 1995) as well as subsequent food bolus disintegration rates in the stomach (Norton, Moore et al. 2007, Kong and Singh 2008, Skamniotis, Elliott et al. 2017). Specifically, oral processing increases the overall surface to volume ratio of the ingested foods to ensure sufficient bolus hydration and enzymatic disintegration rates in the stomach (Bornhorst and Singh 2012); these activities, in turn, assist in the reduction of the food bolus particles sizes at levels small enough for efficient nutrient absorption by intestinal walls (Tharakan, Norton et al. 2010). As a result, failure to shallow sufficiently small particle sizes can lead to maldigestion (Pera, Bucca et al. 2002). This is a delicate matter in pets due to the pronounced variation in masticatory characteristics and consequently chewing capabilities between species (Luke and Lucas 1983, Berthaume, Dumont et al. 2013, Berthaume 2016, Skamniotis, Patel et al. 2016). For this reason, the degree of optimisation in formulating marketable products that match the respective masticatory needs of different pet breeds may still be limited.

Methods of enhancing palatability so far include adjusting moisture and meat content (Gierhart and Hogan 1993, Tran, Hendriks et al. 2008), employing expansion techniques to alter density (Axelrod 1993), calibrating the extrusion parameters (Moscicki 2011) as well as incorporating flavours and other additives (Nahm Jr 1982, Axelrod 1993). However in most processes, altering texture without compromising nutritional value and balance remains a challenge. This creates the need to understand and quantify the effect of various essential nutrients/components on texture itself (Bone and Shannon 1975, Axelrod 1993, Gierhart and Hogan 1993). Some general trends have been established i.e. meats and animal derivatives generally increase palatability (Balaz, Bone et al. 1976, Nahm Jr 1982). However, these only concern a few popular constituents and the relevant literature is limited. Furthermore, such correlations are mostly derived via empirical methods and do not provide enough information for designing texture while controlling food composition in pets.

While texture evaluation through sensory panels has been commonly applied in human food design (Vandenberghe and Claes 2011, Çakır, Vinyard et al. 2012), such methods are associated with prohibitive time resources and high costs since they would require sensory assessment by the pets. In contrast, instrumental methods are straightforward, highly reproducible (Vandenberghe and Claes 2011) and potentially lead to consistent interpretations (Morren, Van Dyck et al. 2015). However, their results are subject to the prescribed test conditions and material parameters (Goh 2002) used; these are often empirically selected and may not relate well with the multiple stimuli and consumer-food interactions in-vivo (Szczesniak 1990, Morren, Van Dyck et al. 2015). On the other hand, fundamental tests measure strictly defined mechanical properties (Morren, Van Dyck et al. 2015) that are major determinants of texture i.e. stress versus strain (stress-strain) response, fracture toughness and viscosity (Goh 2002, Barrangou, Daubert et al. 2006, Chen 2009, Claes, De Maesschalck et al. 2012). The stress-strain property provides a measure of food stiffness (rigidity), expressed as the amount of stress required to achieve a given deformation (strain) level. On the other hand, toughness determines the resistance against fracture, defined as the amount of mechanical energy dissipation, locally at the crack tip, required per unit crack growth surface area. 
The above parameters are size and geometry independent, rendering the fundamental tests ideal for comparative studies between food compositions towards quantifying the effect of various pet food components on texture; they also provide the necessary material property data used as key input parameters in computational models of oral processing, as recently presented by the authors in (Skamniotis, Patel et al. 2016, Skamniotis, Elliott et al. 2017). Conclusively this study is concerned with such fundamental tests

The wire cutting test has been used to determine fracture toughness in foods, although only soft structures were involved, such as starch gels (Gamonpilas, Charalambides et al. 2009) and gelatine gels (Forte, D'Amico et al. 2015). Instead, the Essential work of Fracture (EWF) test has proven a viable solution for obtaining accurate fracture toughness values in a wide range of polymers and particularly in soft bio-polymeric composites (Skamniotis, Kamaludin et al. 2017). Taguet et al. (Taguet, Bureau et al. 2014) employed the EWF test to investigate the toughening effect of glycerol content in thermoplastic starch particles blended with high density polyethylene (HDPE). Ali et al. (Ali, Unnikrishnan et al. 2013) found increasing toughness and ductility with increasing potato starch content in polymethyl-methacrylate (PMMA) via EWF and tensile tests. Chaléat et al. (Chaléat, Halley et al. 2008) used these tests to study the effect of moisture content on the tensile and toughness properties of chemically modified maize starch.

Nevertheless, these studies served in optimising the biodegradability of polymers to address ecological concerns and no relevant literature has been found in edible starch blends. Commercial starch based pet foods are becoming increasingly popular due to convenience in use by the pet owners, reasonable price and nutrient content. Indeed, recent research has found that domesticated pets thrive on a diet rich in starch (Axelsson, Ratnakumar et al. 2013). For this reason, only recently the authors utilised the EWF and cutting methodologies to study fracture in starch based foods (Skamniotis, Patel et al. 2016). Yet, in our previous study only one recipe was considered. In this work we now study the effect of commonly used components by the pet food industry, namely natural fibres and minerals, on the mechanical properties of food products. Cellulose fibres are generally known for their stiffening effects in starch matrices (Wan, Luo et al. 2009) and have been widely used in packaging applications (Sorrentino, Gorrasi et al. 2007). The impact of mineral components on the physicochemical properties of starch e.g. enzymatic hydrolysis, waterbinding capacity and solubility has been also studied (Pietrzyk, Fortuna et al. 2013). Yet, no information currently exists on the effect of such ingredients on the stress-strain and toughness properties of starch food extrudates.

At the same time both fibres and minerals are particularly important in pet food design, not at least in the global food industry. Not only their nutritional value displays an ongoing interest but also their contribution to texture raises concerns (Nahm Jr 1982). Minerals, often declared as 'inorganic matter' on commercial food products typically include (Nutrition 1974, Hand, Hefferren et al. 1995): calcium, phosphorous, magnesium, potassium, sodium and chloride, iron, copper, manganese, zinc, iodine, and selenium. These display different functionalities (Lepine and Reinhart 1998) working together to coordinate various body functions and maintain normal activities on a daily basis such as the formation of bone and cartilage (Lepine and Reinhart 1998), nerve and muscle function, fluid balance regulation, the transportation of oxygen in the bloodstream and hormone production (COUNCIl 1985). On the other hand, fibres are complex carbohydrates commonly found in pet foods (Nutrition 1974, Nahm Jr 1982) as rice hulls, corn, soybean hulls, beet pulp, dried potato product, cellulose, bran, peanut hulls, and pectin. Adding fibre to a diet has been found to improve colon health (Reinhart 1997), i.e. helping to guard against diarrhea and constipation (Reinhart 1997) as well as preventing the overgrowth of harmful 
bacteria. Fibres also assist in the dietary management of obesity by enhancing the feeling of fullness (Norton, Moore et al. 2007, German, Holden et al. 2010).

Our present study for the first time investigates the stiffening and toughening effects of such fibre and mineral components on commercial starch based pet foods. A description of the material compositions studied and experimental methods applied is firstly provided followed by the presentation and discussion of the tensile, EWF and cutting test data; microstructural SEM observations also support the interpretation. Finally, the potential for optimising pet food texture through controlling composition is discussed.

\section{Materials and methods}

\subsection{Materials}

The starch based food specimens were cut from extrudates produced by Mars Petcare, UK, including rectangular (sheet) and square cross sectional profiles; 60 specimens were prepared for uniaxial tensile tests (Figure 1a), 100 specimens were prepared for the EWF tests (Figure 1b) and 42 specimens for the cutting tests (Figure 1c). The varied food constituents which were fed into a twin screw extruder included cereal starch of 15-20 $\mu \mathrm{m}$ raw granule size, water, cellulose fibres of 200-500 $\mu \mathrm{m}$ length and 20-30 $\mu \mathrm{m}$ diameter and minerals in the form of a pre-combined powdered mix of $50 \mu \mathrm{m}$ average particle diameter. The raw mineral ingredients included sodium tripolyphosphate, zinc sulphate, potassium, calcium and phosphorous. Four compositions (in terms of w/w) were extruded; for clarity these are referenced throughout as:

- starch: $50 \%$ starch, $50 \%$ water,

- $\quad$ starch \& fibre: $48.75 \%$ starch, $48.75 \%$ water, $2.5 \%$ fibres,

- $\quad$ starch \& mineral: $47.5 \%$ starch, $47.5 \%$ water, $5 \%$ minerals.

- $\quad$ starch \& fibre \& mineral: $46.25 \%$ starch, $46.25 \%$ water, $2.5 \%$ fibres, $5 \%$ minerals,

The above fibre and mineral concentrations reflect typical numbers used in commercial pet food recipes, while the four blends consistently involved equal masses of starch and water. Potential nozzle size effects on texture between the rectangular and square extrudate profiles were minimised through using nozzles of consistent cross sectional area, $A_{o}=150 \pm 2.1 \mathrm{~mm}^{2}$ (Skamniotis, Elliott et al. 2017), while an extrudate temperature of $90^{\circ} \mathrm{C}$ at the point of die exit and extrusion specific mechanical energy of $60 \mathrm{Wh} / \mathrm{kg}$ were applied throughout. Both the raw extrudates and specimens were rigorously kept sealed after production until testing, to preserve the water content (Skamniotis, Patel et al. 2016). The longer term pronounced ageing effects through starch re-crystallisation were also minimised via conducting all the tests on four consecutive days, three days after production. All the material was stored and tested at a constant ambient temperature of $20{ }^{\circ} \mathrm{C}$ and relative humidity of $50 \%$, controlled by a laboratory airconditioning system. A sensor was also used to monitor potential temperature and humidity gradients within the laboratory room; these were found negligible. 


\subsection{Microscopy analysis}

Scanning Electron Microscopy (SEM) was performed via a Hitachi S-3400N equipment. The latter enabled in-situ SEM imaging, such that the microstructural evolution could be captured while deforming/fracturing the material with the aid of a micro-tester with serrated tensile grips (200 N load cell capacity). This involved comparing SEM images of progressive crack tip opening at the onset of crack propagation between the 'starch \& fibre' and 'starch \& fibre \& mineral' compositions in order to highlight different toughening mechanisms. Small Double Edge Notched Tensile (DENT) specimens of dimensions $15 \mathrm{~mm}$ x $12 \mathrm{~mm}$ x $3 \mathrm{~mm}$ (length $\mathrm{x}$ width $\mathrm{x}$ thickness) were used; these were coated with a gold layer prior to imaging. The study also included images of the DENT specimen post-fractured surfaces (discussed later in Section 3.2), as well as prefracture and post-facture observation of the gauge length material from the uniaxial tensile tests (Figure 1(a)).

The extruded blend displayed an amorphous matrix due to starch gelatinisation (Skamniotis, Patel et al. 2016) with detectable fibre components in contrary to the minerals which were impossible to observe. The latter suggests some degree of mineral dissolution during extrusion cooking (Wani and Kumar 2016), although this was not rigorously verified via the common dry ashing technique described in (Chapman and Pratt 1982); instead, this study focuses on the macroscopic composition effect on the mechanical behaviour.

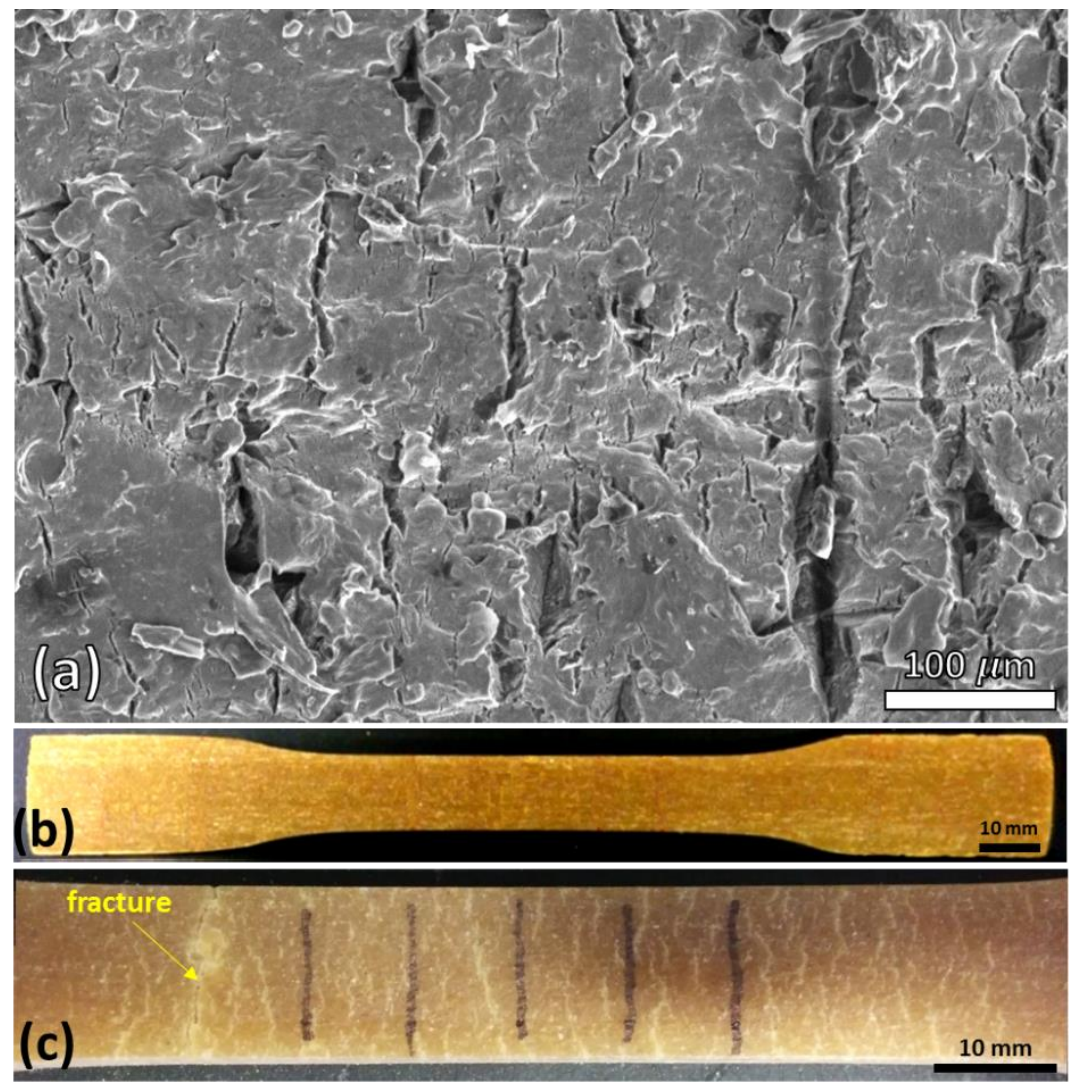

Figure 1. (a) Micro-cracking detected via SEM, (b) undeformed dumbbell specimen used for uniaxial tension cut from sheet profile extrudates, (c) profound micro-cracking along the gauge length of a fractured specimen - fracture occurs through a macro-crack. 


\subsection{Uniaxial tension}

Tensile tests served in understanding and quantifying the composition effect on stiffness and ductility, the latter represented by the tensile failure strain, $\varepsilon_{f}$. All the tests were performed via a single column Zwick Roell universal testing machine with a $1 \mathrm{kN}$ load cell capacity, serrated tensile grips and dumbbell specimens (see Figure 1(b)); these were cut from sheet profile extrudates via a standard specimen die and a mechanical press. The specimen thickness, width and grip to grip dimensions were approximately $5 \mathrm{~mm}, 12 \mathrm{~mm}$ and $100 \mathrm{~mm}$, respectively, while reference lines were drawn along a gauge length of $40 \mathrm{~mm}$ (see Figure 1(c)) and optically monitored during the tests to evaluate strain. Data were collected until ultimate specimen separation for the three constant true strain rates, $\dot{\varepsilon}$, of $0.1 / \mathrm{s}, 0.01 / \mathrm{s}, 0.001 / \mathrm{s}$; these were also used to fit an empirical constitutive power law as described in following section 2.3. Five repeats were performed per test condition; this number has been shown to provide a representative sample in foodstuffs within single batches (Gamonpilas, Charalambides et al. 2009, Mohammed P. Afandi, Charalambides et al. 2013, Skamniotis, Elliott et al. 2017). The data will be plotted throughout as average stressstrain curves and percentage deviation values will be reported; error bars will not be provided to assist in clear illustration and comparison of the curves, but these showed a maximum $\pm 9 \%$ error. Based on the fully incompressible behaviour already found for starch (Poisson's ratio of $v=0.5$ ) in (Skamniotis, Patel et al. 2016), the true (Cauchy) stress, $\sigma$, versus true (Hencky) strain, $\varepsilon$, data were calculated via:

$$
\left.\begin{array}{cc}
\text { (i) } & \sigma=F / A_{i} \\
\text { (ii) } \varepsilon=\ln \left(g_{i} / g_{o}\right)
\end{array}\right\}
$$

where $F$ is the respective applied force, $A_{i}$ is the deformed (current) specimen cross sectional area and $g_{o}, g_{i}$ are the original and deformed specimen gauge lengths, respectively. Note that $A_{i}$ and the original specimen cross sectional area, $A_{o}$, are related through $A_{i} g_{i}=A_{o} g_{o}$, based on the incompressibility assumption.

\subsection{Constitutive power law model}

The tensile response was characterised through fitting a constitutive power law, commonly used in viscoelastic materials (Schiessel, Metzler et al. 1995) and specifically foods such as cheese (Goh, Charalambides et al. 2005). The law postulates that the dependencies of stress upon the applied strain and time are separable, which is true when iso-strain (isometric) plots obtained from stress-relaxation data at different constant strains, are parallel. This was verified here based on relaxation data previously reported in starch (Skamniotis, Kamaludin et al. 2017). The power law is expressed as: 


$$
\sigma=\Phi \varepsilon^{m}\left(\frac{t}{t_{r}}\right)^{-n}
$$

where $\Phi$ is a constant associated with material stiffness with units of elastic modulus (MPa), $m$ is the strain dependent exponent, $n$ is the time dependent exponent and $t_{r}$ is a time constant with the value of $1 \mathrm{sec}$. The concept of each of the parameter is as follows. $\Phi$ scales positively the overall stresses and hence it is used to quantify the overall stiffening effect of fibres and minerals. $m$ describes how the material stiffness and damage mechanisms evolve with increasing strain between the four compositions. It therefore depends on the resistance of the starch matrix to deformation as well as on the extend of microstructural phenomena with increasing strain, such as starch fibre-matrix de-bonding and micro-cracking. As a result, $m$ describes the shape of the stressstrain curves between the four compositions e.g. high and low $m$ values respectively imply material stiffening (rubberlike behaviour) and softening with increasing strain. Finally, $n$ expresses the overall amount of time dependency, or equivalently, the overall significance of the viscous component during deformation between the four compositions. High and low $n$ values respectively associate with increasing and decreasing the range of the stress-strain response as a function of strain rate.

The $\Phi, m$ and $n$ parameters were calibrated through the Microsoft Excel solver macro where the model (power law) predictions (Equation (2)) were matched with the test data via the least squares fit procedure (Mohammed P. Afandi, Wanigasooriya et al. 2017). This involved minimizing the relative absolute error, er, over $n$ data points, between the model stress, $\sigma_{m}$, and experimental stress, $\sigma_{\text {exp }}$, expressed as:

$$
e r=\sum_{i=1}^{n}\left(1-\left|\frac{\sigma_{m}}{\sigma_{\text {exp }}}\right|\right)^{2}
$$

Specifically, the minimisation was performed through the Generalized Reduced Gradient (GRG) Nonlinear engine, where the three constants were set to vary until er converges to an improved value i.e. er does not change significantly within ten iterations.

The aim of fitting the power law is to allow accurate reproduction of the stress-strain data presented here by other authors in order to compare between results obtained from different studies. In addition, since the $\Phi, m$ and $n$ parameters are independent of the experimental conditions i.e. strain and strain rate, they can be used to compare the constitutive response of various food matrices. Here the power law is used to quantify the stiffening effects of fibres and minerals via calibrating the parameters firstly for the 'starch' composition and then calibrating $\Phi$ for the other compositions while keeping $m$ and $n$ fixed. By this way, the model fit results will indicate (shown later in section 3.1) whether the effect of composition can be described only through a single parameter, $\Phi$, such that the stress-strain shape and rate dependency are not influenced by the inclusion of fibres and minerals. 


\subsection{Fracture experimental methodologies}

The EWF and cutting tests are shown to be suitable methods for deriving accurate toughness values in foods (Skamniotis, Patel et al. 2016, Skamniotis, Kamaludin et al. 2017). This is because foods, like other highly dissipative polymers, do not obey the Linear Elastic Fracture Mechanics (LEFM) requirements. Specifically, the energy release rate, $G_{c}\left(\mathrm{~kJ} / \mathrm{m}^{2}\right)$, also known as fracture toughness or essential work of fracture, $w_{e}$, cannot be calculated in a straightforward manner, i.e. through the area under the force-displacement curve of a cracked body (Williams 1984). This is because $G_{c}$ is meaningful when it is measured as the energy per unit crack growth area dissipated locally at the crack tip, whereas the area under the force-displacement curve of a cracking starch specimen carries the contribution of energy dissipation due to material deformation non-essential (non-local) to the crack tip (viscous, plastic and/or micro-cracking effects).

The concept of the EWF and cutting methods is similar in that both separate the total energy into that involved in creating new crack faces (local/essential) and the non-essential energy dissipated through remote material deformation; cutting also involves frictional dissipation between the cutter and the sample. These energy components are decoupled based on the following energy conservation principles:

EWF: $\quad w_{f}=w_{e}+w_{p} \beta l$

$$
\text { Cutting: } \quad\left(F_{c}-F_{t}\right) \frac{\tan \phi}{b}=G_{c}+\frac{\sigma_{Y}}{2}\left(\tan \phi+\frac{1}{\tan \phi}\right) h
$$

where in Equation (4), $l$ is the specimen un-cracked (ligament) length (see Figure 2(a)), $w_{f}$ is the total energy normalised over the ligament specimen cross sectional area (specific total work), $w_{p}$ is the energy consumed per unit plastic/inelastic volume (specific non-essential work of fracture) within a specimen region determined by the plastic/inelastic zone shape factor, $\beta$, and $w_{e}$ is the energy consumed per unit fractured ligament area, known as the essential work of fracture, equivalent to $G_{c}$. The term $\beta$ is a material parameter that indicates the shape of the plastic/inelastic zone; here for simplicity a circular zone shape is used, leading to $\beta=\pi / 4$, while recent study (Skamniotis, Kamaludin et al. 2017) highlights that the value has no influence on the $w_{e}$ result derived through the EWF method.

In Equation (5), $h$ and $b$ are the cut depth and width, $F_{c}$ is the major cutting force applied on the blade along the cutting direction accompanied by the vertical $F_{t}$ force generated due to the cut chip-tool interface conditions and $\phi$ is the angle of the shear plane responsible for plastic deformation on the chip according to a Tresca yield stress criterion, $\sigma_{Y}$ (see Figure 2(b) for symbols) (Williams and Patel 2016). Equation (5) assumes that the cutting speed is sufficiently low such that no kinetic energy is involved, as well as that the cutting process is isothermal such that tool-chip friction and material energy dissipation processes does not lead to temperature changes. Although the latter may not hold for metal cutting processes especially at high cutting speeds, it has been established as a reasonable assumption in soft biological systems (Williams and Patel 2016). The equivalency between Equations (4) and (5) and thus the EWF and cutting methods, respectively, lies in the fact that both imply a linear equation, $y=y_{1}+\lambda x$, where $y$ represents total energy involved in the process ( $w_{f}$ in EWF and $\left(F_{c}-F_{t}\right) \frac{\tan \phi}{b}$ in cutting), the slope, $\lambda$, relates to the non-essential energy dissipation density $\left(w_{p} \beta\right.$ in EWF and $\frac{\sigma_{Y}}{2}\left(\tan \phi+\frac{1}{\tan \phi}\right)$ in 
cutting), the variable, $x$, associates with the geometry of the initial crack/cut ( $l$ in EWF and $h$ in cutting) and the intercept, $y_{1}$, denotes surface energy dissipation to open new crack faces i.e. fracture toughness ( $w_{e}$ in EWF and $G_{c}$ in cutting).

Therefore, via performing a set of EWF tests in which $w_{f}$ is measured for specimens of different ligament length, $l$, the $w_{e}$ and $\beta w_{p}$ are determined by linear regression on $w_{f}$ versus $l$ data; equivalently via measuring $\left(F_{c}-F_{t}\right) \frac{\tan \phi}{b}$ for a set of different cut depths, $h$, the $G_{c}$ and $\frac{\sigma_{Y}}{2}\left(\tan \phi+\frac{1}{\tan \phi}\right)$ are found by linear regression on $\left(F_{c}-F_{t}\right) \frac{\tan \phi}{b}$ versus $h$. The derivation of Equation (4) and a further description of the EWF and cutting theories are provided in (Skamniotis, Patel et al. 2016) and (Williams and Patel 2016).
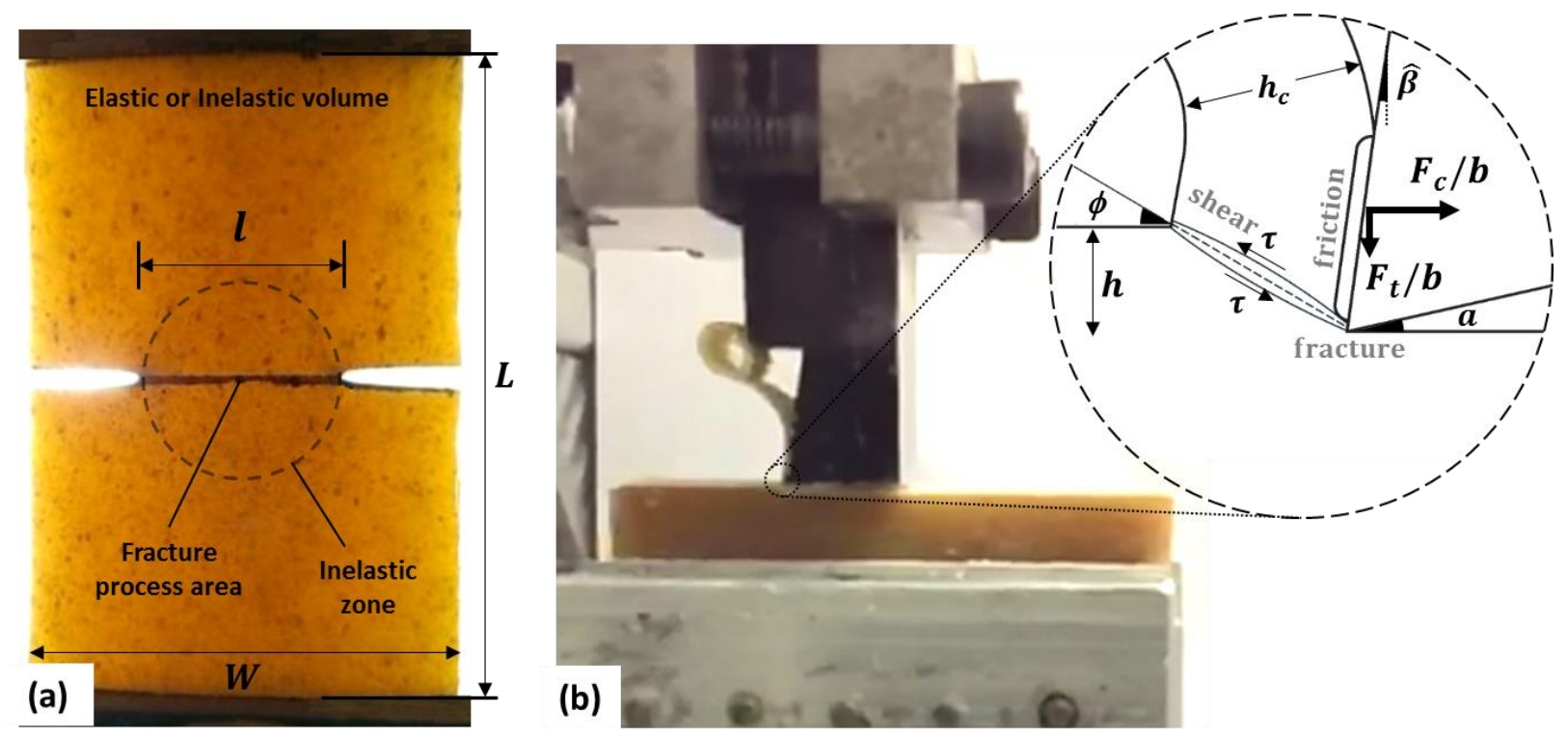

Figure 2. Demonstration of the EWF and cutting tests: (a) DENT specimen with ligament length, $l=14 \mathrm{~mm}$, used in EWF tests cut from sheet profile extrudates; three distinct process zones are denoted (c) chip formation during cutting on square profile extrudates and schematic of the analysis performed at the cutting tip.

\subsection{EWF}

The DENT specimen configuration shown in Figure 1(b) was obtained via cutting long sheet profile extudates of thickness $B=4.9 \pm 0.56 \mathrm{~mm}$ and width $W=29 \pm 1.1 \mathrm{~mm}$ into standard lengths of $L=50 \mathrm{~mm}$. Thereafter notches were generated via pushing razor blades held in a manual press which allows for fine displacement control; a new razor was used for each cut. To ensure notch alignment the specimen was fixed in a drill vice while a line marked across the specimen width assisted in guiding the blade (Saleemi and Nairn 1990). Specimens with misaligned notches were discarded. The machine (crosshead) displacement data were judged adequate to facilitate comparison between the four recipes; thus no additional gauge length measurements were performed in order to correct for the diffuse energy dissipation effects reported in (Skamniotis, Kamaludin et al. 2017). Five original ligament lengths of 6, 8, 10, 12, $14 \mathrm{~mm}$ were tested using five replicates per condition. The standard error calculation for $w_{e}$ and $\beta w_{p}$ was based on the method described in (Marchal, Walhin et al. 1997). The ligament range was selected to give 
rise to commonly used plane strain condition, $l<3 \mathrm{~B}$, as well as the criterion against large deformations in the sample arms, $l \leq W / 2$; these criteria have been used for starch and discussed in detail in (Skamniotis, Kamaludin et al. 2017). A crosshead speed, $\dot{\delta}$, of $100 \mathrm{~mm} / \mathrm{min}$ was applied.

\subsection{Cutting}

Cutting was performed along the surfaces of square profile extrudates as shown in Figure 1(c) of the 'starch \& fibre', 'starch \& mineral' and 'starch \& fibre \& mineral' compositions. Conducting additional tests on the 'starch' recipe was not judged necessary in order to assess the validity of the cutting test in fibre reinforced matrices and investigate its equivalence with the EWF test. The extrudates had a sufficient length, $L=50 \mathrm{~mm}$, to provide a steady-state cutting condition and a sufficient width of $b=10 \pm 0.3 \mathrm{~mm}$ to ensure a plane strain condition. Initial cuts were performed on the raw surfaces to obtain a smooth flat reference surface. The blade was made of tool steel with dimensions: width, $b_{1}=12.5 \mathrm{~mm}$, rake angle, $a=10^{\circ}$, and clearance angle, $\hat{\beta}=11^{\circ}$. The tip radius was measured approximately as $\rho=5 \mu \mathrm{m}$ before and after tests via an optical microscope to ensure there was no tip blunting; this value has been reported sharp enough to give $G_{c}$ independent of radius in tough materials such as starch (Blackman, Hoult et al. 2013). Fourteen cut depths were chosen with values uniformly lying in the range $0.15<h<0.4 \mathrm{~mm}$; these were controlled via moving the specimen stage upwards to the desired plane and measuring the position of the surface before and after each cut through a dial gauge (Mitutoyo UKAS 3533733). The standard error calculation for $G_{c}$ and $\sigma_{Y}$ was based on the method described in (Marchal, Walhin et al. 1997). A tri-axial load cell mounted on the blade tool was used to measure $F_{c}$ and $F_{t}$; an average value in the steady-state cutting regime was used in the analysis (Equation (5)) for each cut. A cutting speed, $\dot{c}$, of $50 \mathrm{~mm} / \mathrm{s}$ was used. Immediately after the tests the cut chip thickness, $h_{c}$, measurements were performed using a micrometer (RS Pro UKAS 7051216); these were necessary to determine the angle, $\phi$, through (Williams and Patel 2016):

$$
h_{c}=\frac{h \cos (\phi+\alpha)}{\sin \phi} \Rightarrow \tan \phi=\frac{\cos \phi}{\frac{h_{c}}{h}-\sin \phi}
$$

\section{Results and discussion}

\subsection{Tensile stress-strain data}

Figure 3 depicts the effect of strain rate, $\dot{\varepsilon}$, on the average experimental tensile stress-strain curves in the four compositions, together with the power law model fit. The same test data are plotted in Figure 4 to highlight the effect of composition for the three strain rates. The failure strain, $\varepsilon_{f}$, values correspond to the last data point of each curve, where ultimate failure occurred. A maximum typical variability of approximately $9 \%$ is observed in the average stress values while a maximum variability of $7.5 \%$ is obtained in $\varepsilon_{f}$. 
Figure 3 displays an excellent fit for the power law in all the compositions, based on consistent material parameters $m=0.615$ and $n=0.147$, and of course a varying $\Phi$ between compositions to account for the stiffening/softening effect. A common $n$ parameter (see legends in Figure 3 ) highlights that practically the fibre and mineral components do not have any effect on rate dependency. All the compositions show a non-linear stress-strain response without an obvious yield point, in agreement to previous studies (Skamniotis, Patel et al. 2016, Skamniotis, Kamaludin et al. 2017). An increasing tensile failure strain, $\varepsilon_{f}$, with strain rate, $\dot{\varepsilon}$, is also profound; this behaviour has been well characterised in recent work (Skamniotis, Elliott et al. 2017) and was attributed to the fundamental dependence of $\varepsilon_{f}$ on both the duration and level of previously applied strain, which are of course a function of $\dot{\varepsilon}$.
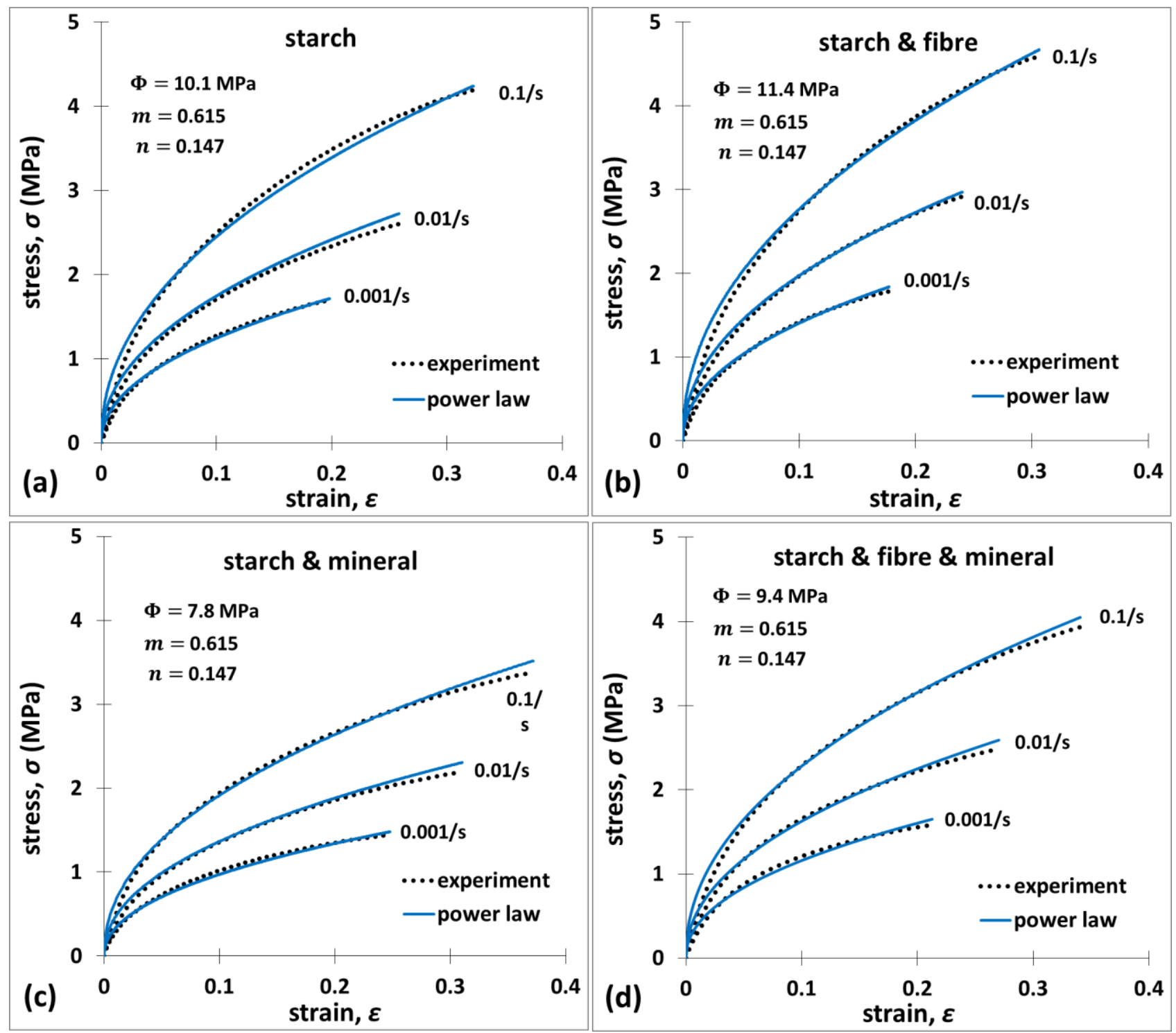

Figure 3. Uniaxial tensile stress-strain response and power law model fit as a function of strain rate in the four compositions: (a) starch, (b) starch \& fibre, (c) starch \& mineral, (d) starch \& fibre \& mineral; the associated power law parameters are denoted. 
On the other hand, Figure 4 shows a noticeable effect of composition on the overall stress levels and ductility in terms of $\varepsilon_{f}$. By comparing stress levels between the 'starch' and the 'starch \& fibre' recipes, as well as between the 'starch \& fibre \& mineral' and the 'starch \& mineral' recipes, the fibre stiffening effect is evident in all the strain rates. However, the inclusion of fibres reduces the strain at break, $\varepsilon_{f}$. These two effects are in agreement with previous studies (Nahm Jr 1982, Moscicki, Mitrus et al. 2013), and are respectively attributed to the stiff and relatively brittle nature of cellulose (Bledzki and Gassan 1999). On the other hand, by comparing the 'starch' against the 'starch \& mineral', and/or the 'starch \& fibre' against the 'starch \& fibre \& mineral' composition, the mineral components appear to increase ductility considerably (reduce stiffness and enhance $\left.\varepsilon_{f}\right)$. 


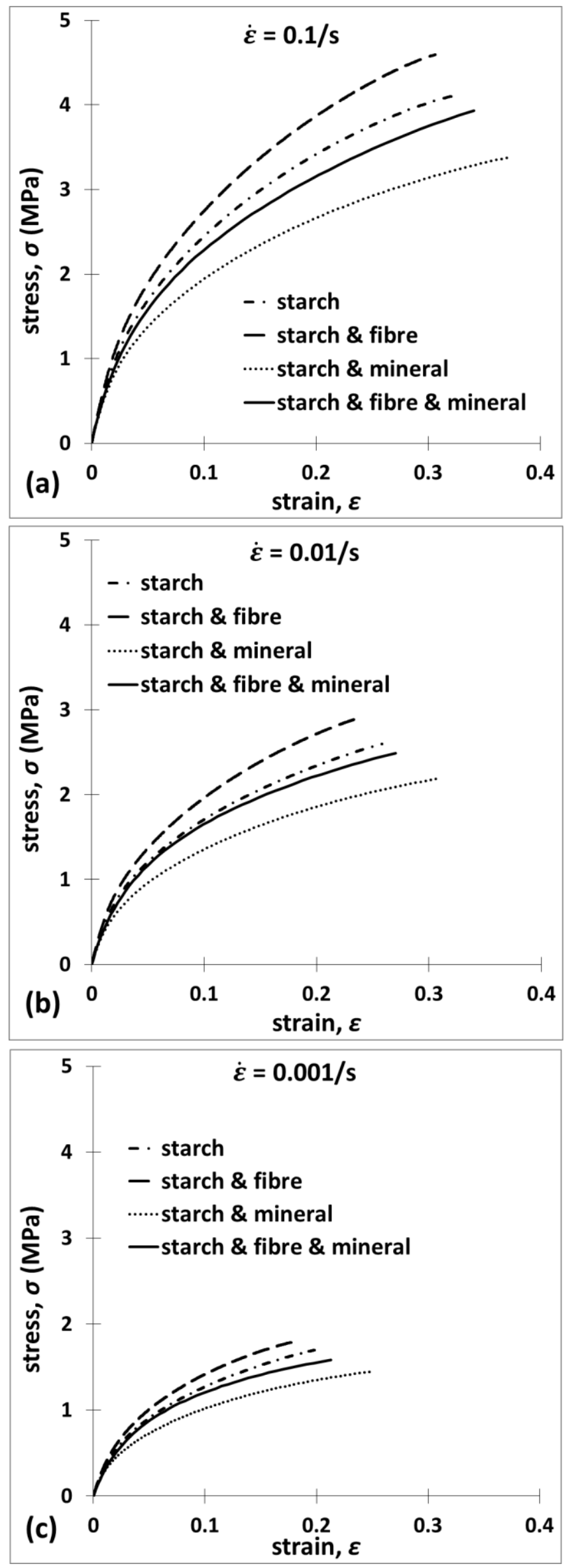

Figure 4. Effect of composition on the stressstrain response for three rates: (a) $0.1 / \mathrm{s}$, (b) $0.01 / \mathrm{s}$, (c) $0.001 / \mathrm{s}$. 
The mechanism that triggers the latter phenomenon is not clear. Owing to a low cellulose fibre surface roughness, mineral-fibre binding and therefore related chemical interactions are unlikely to occur. On the other hand, due to the presence of various mineral ingredients and the unknown mechanical and chemical interaction between these and the matrix, potential plasticisation of the starch structure (Moscicki, Mitrus et al. 2013) and other mechanisms were difficult to investigate. In addition, there is currently no literature on the effect of such components on the mechanical properties of foodstuffs. Based on pervious study on potassium-enriched starch (Pietrzyk, Fortuna et al. 2013), it is likely that also here minerals cause an overall decrease in the thermal stability of starch granules, enhancing water penetration into their interior, increasing chain mobility and finally increasing macroscopic ductility. Furthermore, it should be also noted that the samples of the 'starch \& mineral' and 'starch \& fibre \& mineral' recipes, which included minerals, displayed a clearly lighter brown, uniform colour compared to the other two recipes. The latter supports further the argument that some minerals did dissolve in the starch matrix during extrusion (Section 2.1).

\subsection{EWF \& Microscopy data}

Figure 5 depicts the average force-displacement, $F-\delta$, data for the four compositions; the results are found repeatable with a typical maximum variability less than $8 \%$ in the area computed under the curves. All compositions satisfy the common 'self-similarity' criterion which requires that the $F-\delta$ curves between varying ligaments, $l$, are similar; the latter adds validity to the method (Skamniotis, Patel et al. 2016). Clearly, the 'starch \& fibre' composition (Figure 5(b)) displayed profoundly higher forces compared to all the other compositions (Figures 5(a),5(c),5(d)), in agreement to the corresponding tensile data for these recipes (shown in Figure 4). Such differences are highlighted in Figure 6 where the data used in Figure 5 are re-plotted for the minimum, $l=6 \mathrm{~mm}$ (Figure 6(a)), and maximum, $l=14 \mathrm{~mm}$ (Figure 6(b)), ligaments individually. Specifically, for the 'starch \& mineral' samples, a correlation can be drawn between the increased $\varepsilon_{f}$ found in tension (Figure 4) and the enhanced displacements shown in Figure 6. Similarly, the stiffer but less ductile stress-strain response of the 'starch \& fibre' samples (Figure 4) relate to the higher forces and decreased displacements displayed in Figure 6. The consistent differences in the response of the four compositions, irrespective of ligament length, as well as the agreement with the tensile data added further credibility to the experimental methodology.

An exception is the slightly lower displacements shown by the 'starch' samples compared the 'starch \& fibre' samples, which contradicts the corresponding uniaxial tensile responses. Interestingly, the latter phenomenon is more noticeable with decreasing $l$ (compare Figure 6(a) against 6(b)). It is suggested that the extra displacement associates with the fibre bridging mechanism occurring at the fracture process zone in the 'starch \& fibre' composition, as highlighted by the SEM data in Figure 7. Yet, in Figure 7 it is not clear whether fibre pull-out or fibre breakage is the dominant mechanism. Instead, SEM observations of the fractured sample faces between the 'starch $\&$ fibre $\&$ mineral' and 'starch $\&$ fibre' compositions, summarized by Figure 8, revealed that these toughening mechanisms compete with each other (Lawrence 1972, Oksman, Mathew et al. 2009, Collyer 2012). Specifically Figure 8(a) shows larger fibre pull-out lengths compared to Figure 8(b), giving 


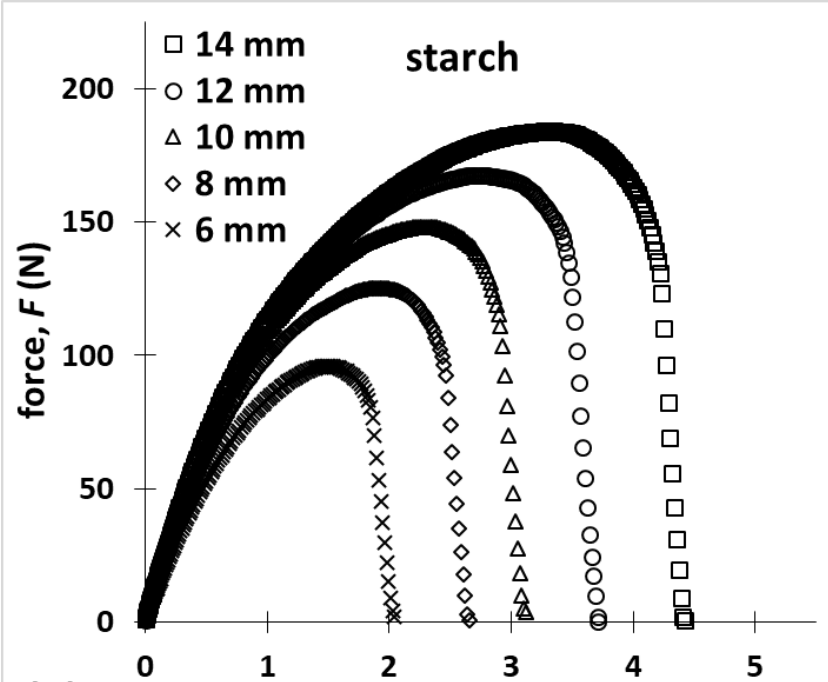

(a) displacement, $\delta(\mathrm{mm})$

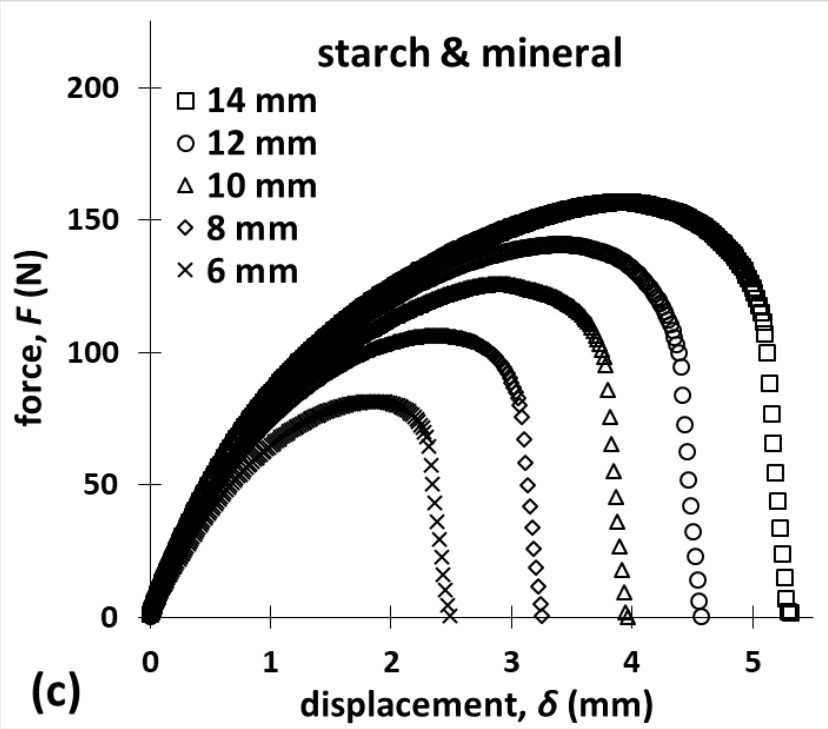

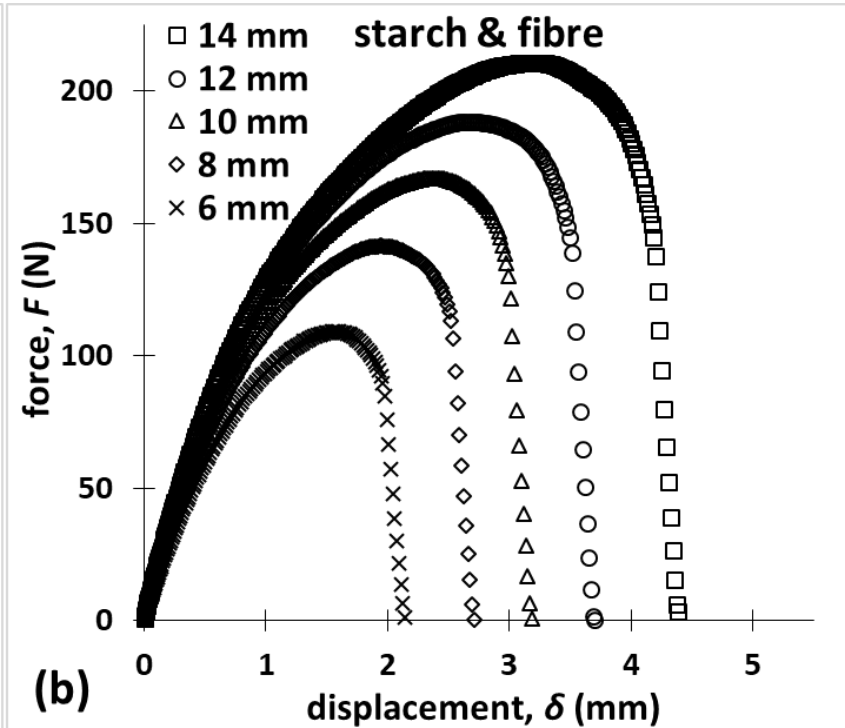

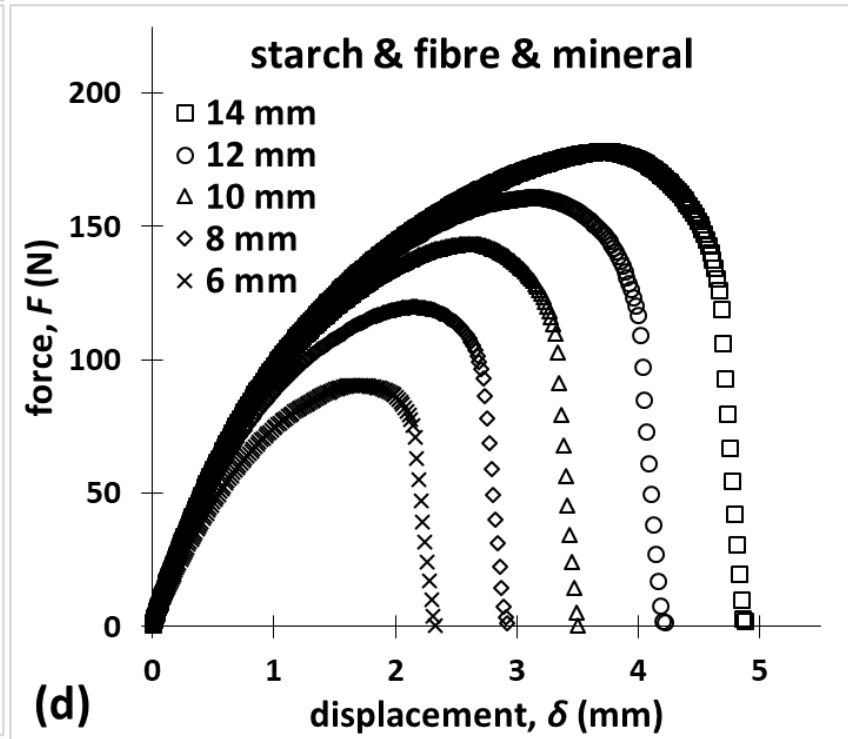

Figure 5. Average force-displacement data for the four recipes: (a) starch, (b) starch \& fibre, (c) starch \& mineral, (d) starch \& fibre \& mineral.
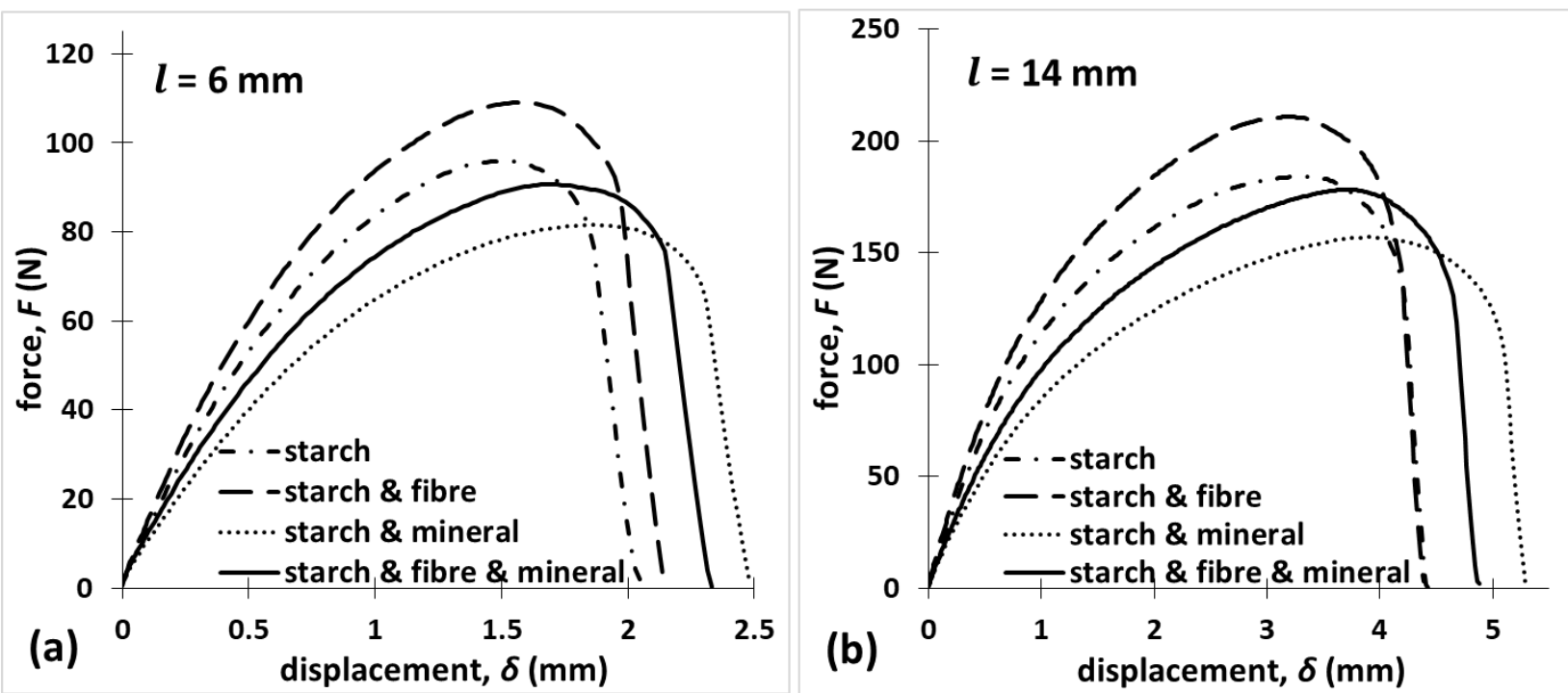

Figure 6. Comparison of the force-displacement curves between the four compositions for the minimum and maximum ligaments: (a) $l=6 \mathrm{~mm}$, (b) $l=14 \mathrm{~mm}$. The behaviour is similar for the other ligaments. 


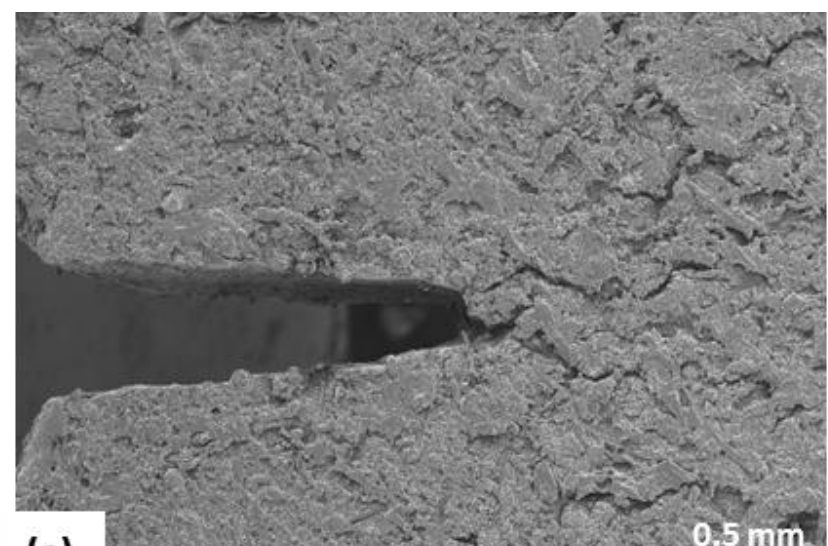

(a)
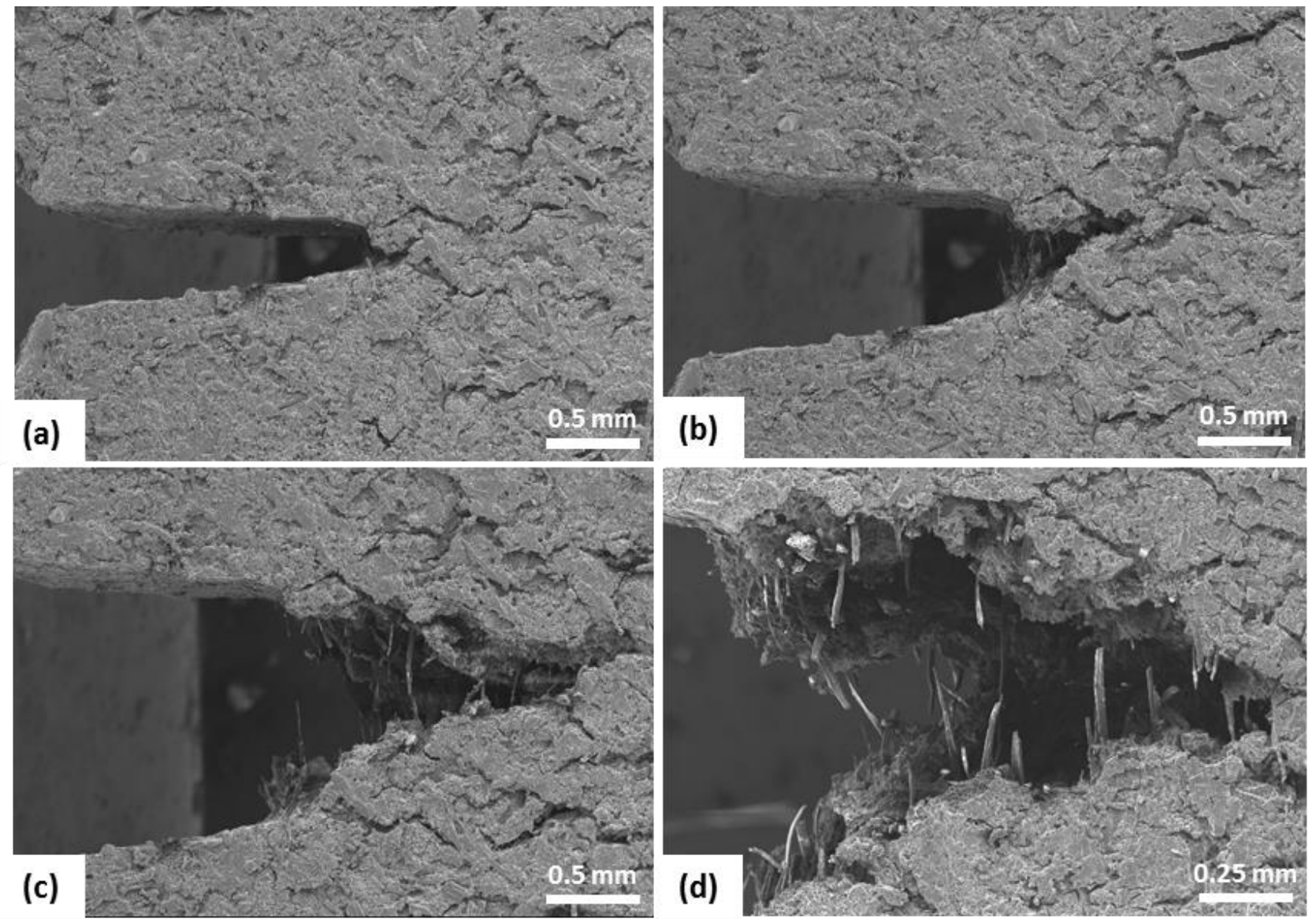

Figure 7. Images captured during in-situ SEM tensile tests on DENT samples of the 'starch \& fibre' recipe; fibre bridging occurs across the crack faces while micro-cracking is present in the crack tip region. SEM parameters: $10 \mathrm{kV}, 60 \mathrm{~mm}, \mathrm{x} 60$.
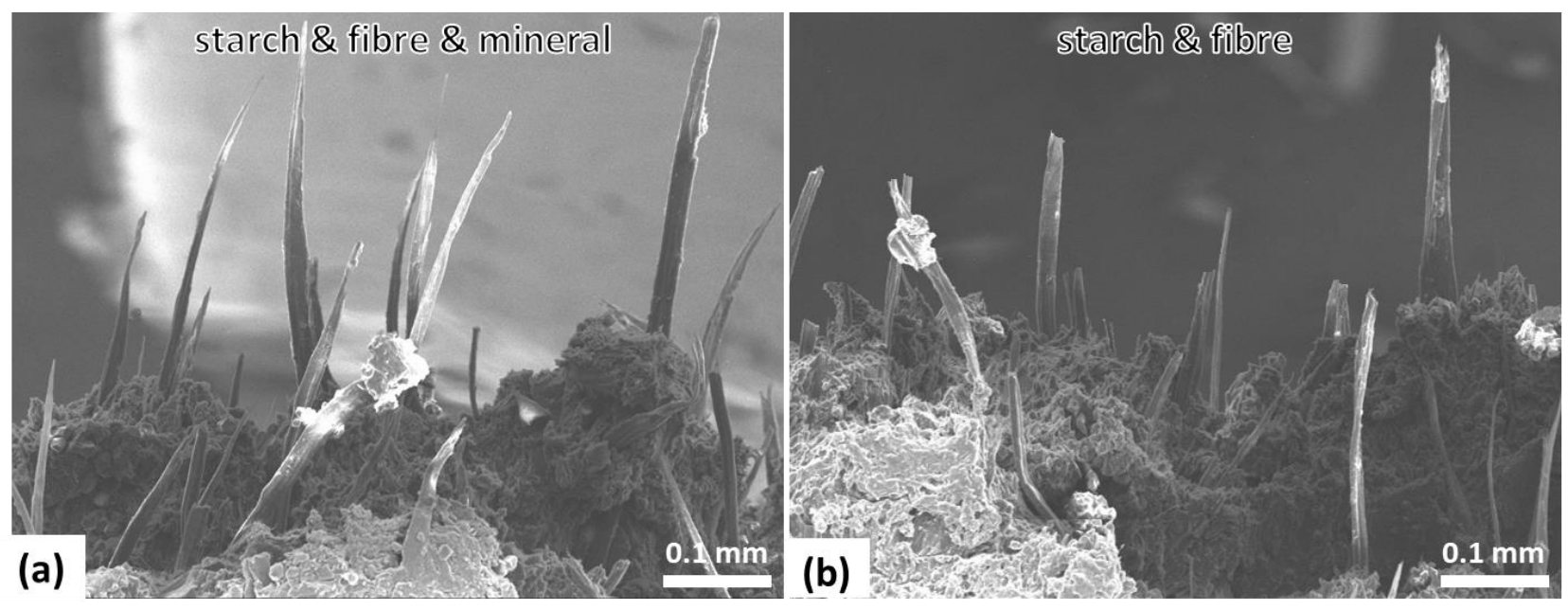

Figure 8. Comparison of the 'starch \& fibre \& mineral' versus the 'starch \& fibre' composition in terms of the dominant fibre toughening mechanism: fibre pull-out or fibre breakage; (a) starch \& fibre \& mineral - average fibre pull-out length $0.21 \pm 0.08 \mathrm{~mm}$, (b)starch \& fibre - average fibre pull-out length $0.15 \pm 0.05 \mathrm{~mm}$. The images show the fractured faces of samples tested via in-situ SEM as shown in Figure 5. 
average values of $0.21 \pm 0.08 \mathrm{~mm}$ and $0.15 \pm 0.05 \mathrm{~mm}$ (measured optically), respectively, and no profound evidence of fibre breakage. Consequently, the fibre pull-out toughening mechanism is rather dominant in the 'starch \& fibre \& mineral' recipe and the inclusion of minerals leads to a $41 \%$ increase in the average fibre pull-out length. In contrast, Figure 8 (b) shows fractured fibre ends suggesting that both fibre breakage and fibre pull-out occur in the 'starch \& fibre' composition.

Both mechanisms have been well characterised for fibre-reinforced composites (Fu, Feng et al. 2008, Collyer 2012), and the occurrence of one over the other is found to depend on various parameters such as the fibre length and orientation, the adhesion properties between the fibres and the matrix as well as the difference in stiffness between fibres and matrix (Bledzki and Gassan 1999). Analytical models that take into account the above properties have been also developed, along with numerical micromechanical models. These approaches however were difficult to implement here owing to the large degree of uncertainty in the final properties of the fibres i.e. elastic modulus and orientation, induced by the extrusion cooking process, not to mention the unknown fibre-matrix interfacial properties. Further challenges are imposed by the strongly nonlinear rate dependent response of starch which of course does not satisfy the common assumption of a linearly elastic matrix upon which such theoretical models rest (Holzapfel and Gasser 2001). Nevertheless, the existing theory (Lawrence 1972, Takaku and Arridge 1973) does lead to a useful interpretation of Figure 6. Specifically, the significantly higher stiffness of the cellulose fibres reported in the literature (Bledzki and Gassan 1999) compared to the starch matrix explains the occurrence of fibre pull-out in both Figures 8(a) and 8(b). This effect is expected to reduce by decreasing the difference in stiffness between fibre-matrix, which is indeed seen here when the softening mineral components are excluded i.e. from 'starch \& fibre \& mineral' (Figure 8(a)) to 'starch \& fibre' composition (Figure 8(b)). In this case, a larger amount of stress is carried by the fibres leading also to fibre breakage phenomena along with fibre pull-out.

The effect of the above mechanisms on fracture toughness, $w_{e}\left(=G_{c}\right)$, is demonstrated in Figure 9, where the EWF extrapolation data based on Equation (4) are compared between the four compositions. The corresponding $w_{e}$ and $w_{p} \beta$ data are also shown in Figures 12(a) and 12(c), respectively, for three compositions to enable comparison with the cutting data later in section 3.4. The toughness results are: $2.92 \pm 0.25 \mathrm{~kJ} / \mathrm{m}^{2}$ for 'starch', $3.59 \pm 0.3 \mathrm{~kJ} / \mathrm{m}^{2}$ for 'starch \& fibre', $3.09 \pm 0.27 \mathrm{~kJ} / \mathrm{m}^{2}$ for 'starch \& mineral' and $3.75 \pm 0.32 \mathrm{~kJ} / \mathrm{m}^{2}$ for the 'starch \& fibre \& mineral' composition. Overall, the inclusion of fibres in the composition does enhance toughness (compare 'starch \& fibre \& mineral' with 'starch \& mineral' and 'starch \& fibre' with 'starch'), while the contribution of minerals alone is very little (compare 'starch \& fibre \& mineral' with 'starch \& fibre' and 'starch \& mineral' with 'starch'). However, the presence of both minerals and fibres gives the maximum $w_{e}$, as the lower slope, $w_{p} \beta$, in the 'starch \& fibre \& mineral' composition compared to the 'starch \& fibre', leads to a higher Y-intercept (toughness). These results indicate that the fibre breakage and fibre pull-out mechanisms together enhance $w_{e}$ further than the fibre breakage mechanism alone. In addition, it was found that the above trend found for $w_{e}$ as a function of composition does not exactly agree with the corresponding trend for the areas under the stressstrain curves. Instead, $w_{e}$ depends specifically on mechanisms occurring at the crack-tip, which may not be revealed via tensile tests alone. In addition, The EWF slope, $w_{p} \beta\left(\mathrm{kJ} / \mathrm{m}^{3}\right)$, is traditionally proportional to the material yield stress (Williams and Rink 2007). However, in this highly dissipative material, where a distinct yield point does not exist, $w_{p} \beta$ can be more accurately related to the material deformation energy density associated with a strain cycle (Skamniotis, 
Kamaludin et al. 2017); this is equivalent to the area under the stress-strain curve. This explains the correlation between the largest area under the tensile response (Figure 4) and the respective highest slope, $w_{p} \beta$, (Figures 9 and 12) for the 'starch $\&$ fibre' composition. This trend is further verified via the following cutting data.

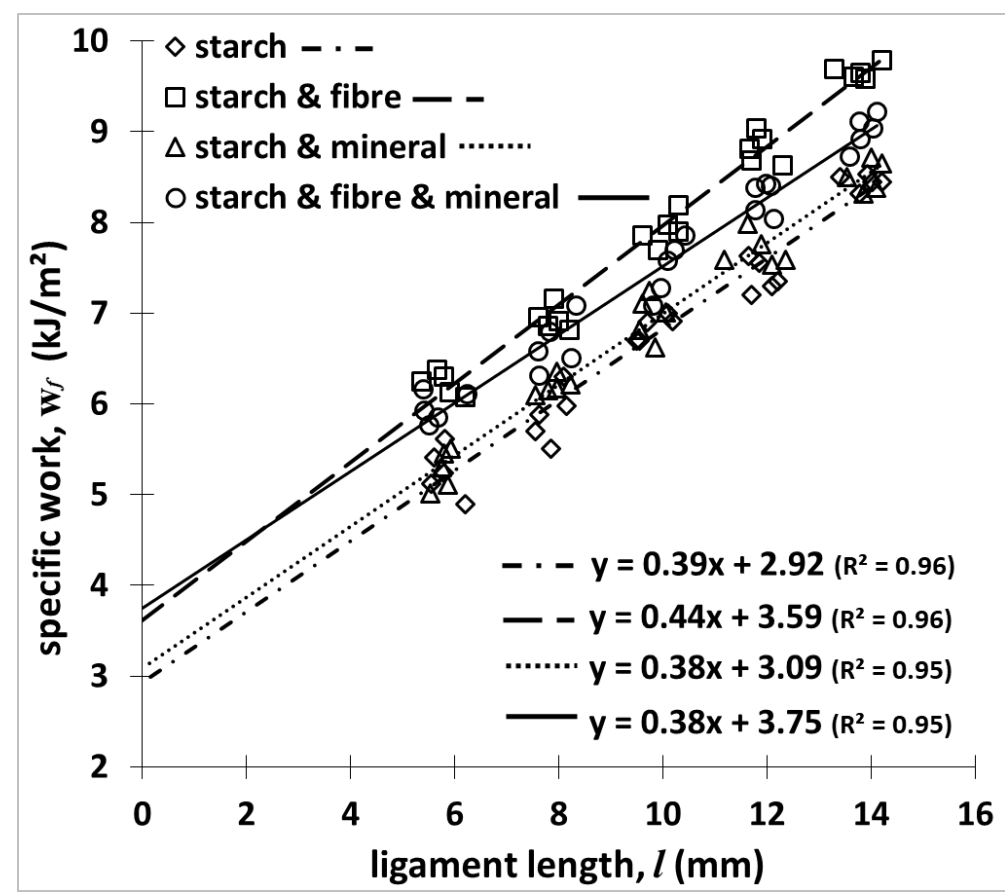

Figure 9. Comparison between the EWF extrapolation data of the four recipes; the linear fits yield $w_{e}$ (intercept) and $\beta w_{p}$ (slope).

\subsection{Cutting data}

Figure 10 summarizes the normalized force over sample width, $F / b$, against cut depth, $h$, data. The apparent linearity in the behaviour verifies the validity of the cutting analysis used via Equation (5). Slightly higher $F / b$ values can be seen for the 'starch \& fibre' (Figure 10(b)) compared to the other recipes (Figures 10(a),(c)), in agreement with the EWF data shown in Figure 6 , as well as the stress-strain curves in Figure 4. Figure 11 illustrates the cutting extrapolation intercept results, being $1.78 \pm 0.13 \mathrm{~kJ} / \mathrm{m}^{2}$ for 'starch \& fibre', $1.74 \pm 0.12 \mathrm{~kJ} / \mathrm{m}^{2}$ for 'starch \& mineral' and $1.87 \pm 0.13 \mathrm{~kJ} / \mathrm{m}^{2}$ for the 'starch \& fibre \& mineral' composition, while the corresponding slopes/yield stresses are $15.6 \pm 0.97 \mathrm{MPa}, 12.26 \pm 0.8 \mathrm{MPa}$ and $13.21 \pm 0.78 \mathrm{MPa}$; these results are also depicted in Figures 12(b) and 12(d), respectively. Again the 'starch \& fibre $\&$ mineral' composition gives the highest toughness (see Figures 11 and 9) and the stiffest 'starch $\&$ fibre' composition indicates the highest slope of 15.6 MPa (also see Figure 12(d)). The latter verifies the equivalency between the EWF parameter, $w_{p} \beta$, and the respective cutting parameter, $\sigma_{Y} \frac{1}{2}\left(\tan \phi+\frac{1}{\tan \phi}\right)$. Note that for illustration purposes Figure 11 embodies the $\frac{1}{2}\left(\tan \phi+\frac{1}{\tan \phi}\right)$ parameter in the $\mathrm{X}$-axis such that the slopes directly correspond to the yield stress, $\sigma_{Y}$. The $\sigma_{Y}$ values (Figures 11 and 12) are higher (by approximately $10 \mathrm{MPa}$ ) but proportional to the stress levels seen in the corresponding stress-strain data between compositions shown in Figure 4. Higher cutting $\sigma_{Y}$ values than the tensile $\sigma_{Y}$ values have been previously found in starch (Skamniotis, Patel et al. 2016) as well as in other polymers and metals (Patel, Blackman et al. 2009, Patel, Blackman et al. 2009). It is attributed to the assumption of the cutting analysis that the material behaves in an 
elastic-perfectly plastic manner (constant yield stress), which fails to predict the true material yield stress, $\sigma_{Y}$, when work hardening occurs (Patel, Blackman et al. 2009) or the stresses continuously increase with strain, such as in Figure 4 here. There is currently no cutting theory that takes into account such behaviours. 

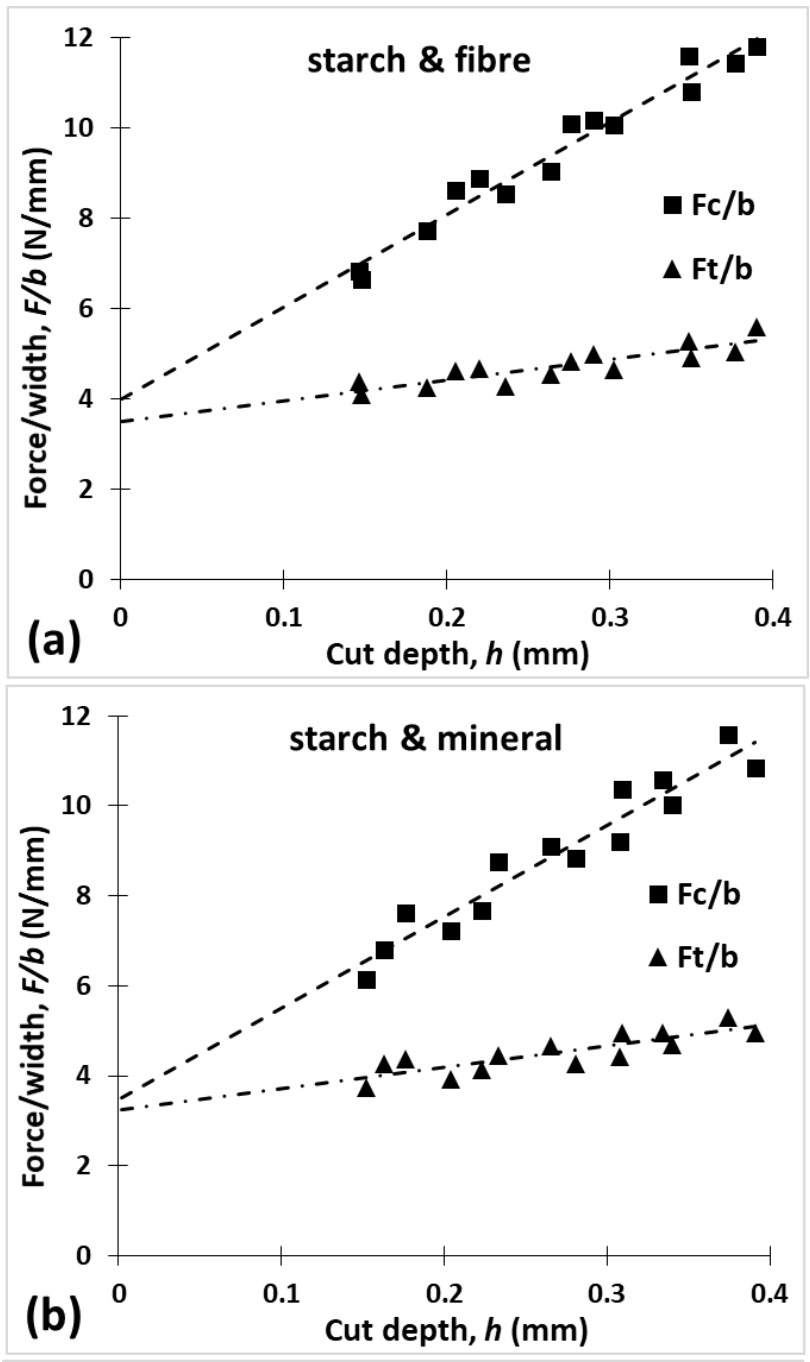

Figure 10. Normalized force over the sample width against the cut depth for the three recipes: (a) starch \& fibre, (b) starch \& mineral, (c) starch \& fibre \& mineral.

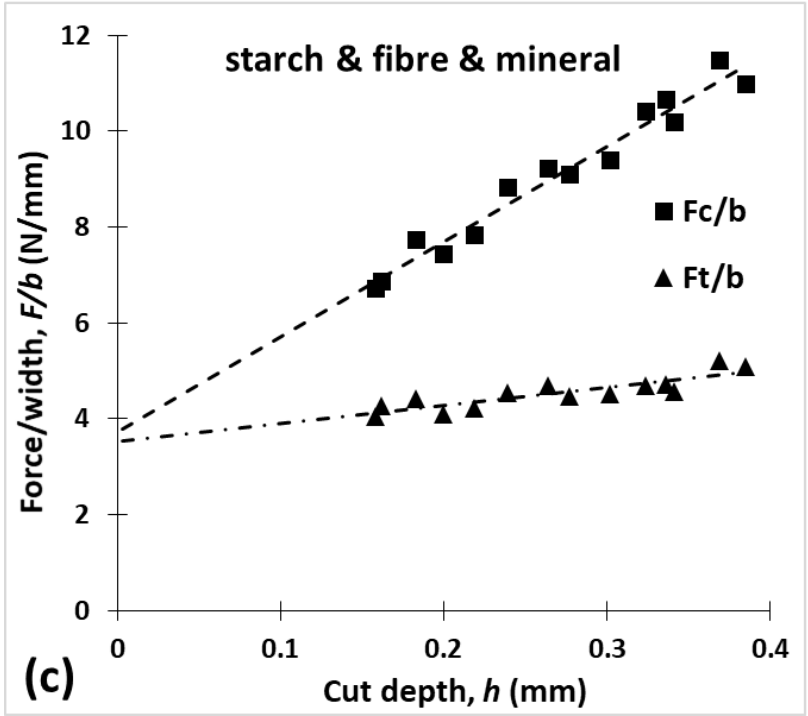




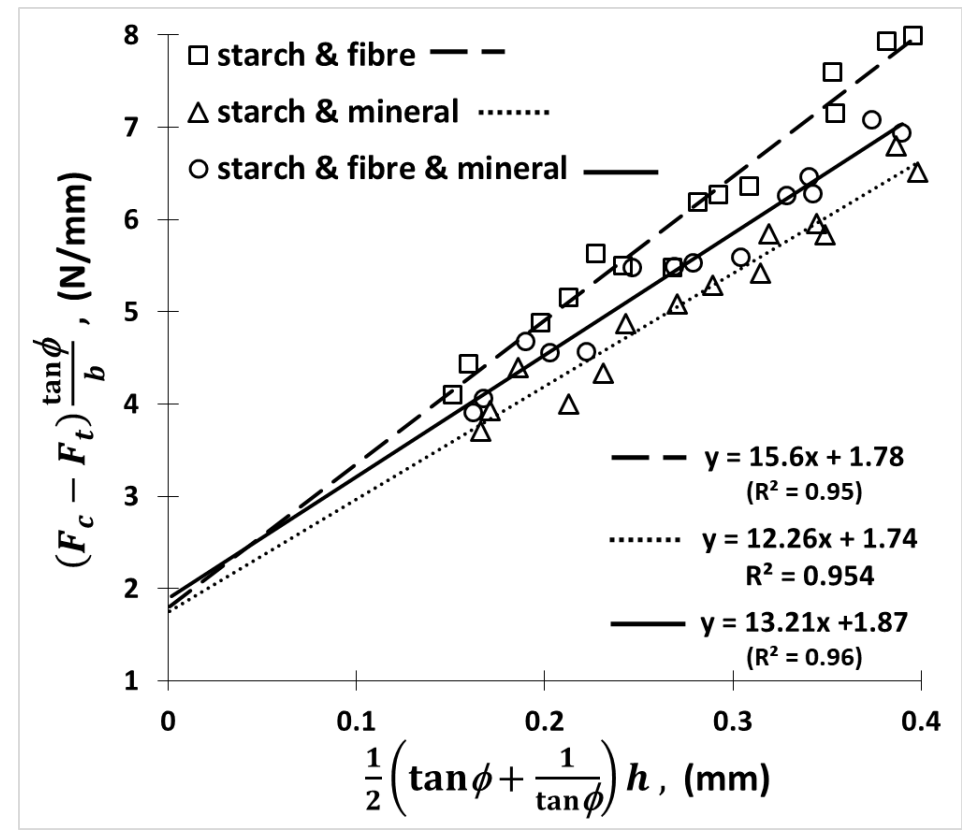

Figure 11. Comparison of the cutting extrapolation data between the three recipes: 'starch \& fibre', 'starch \& mineral' and 'starch \& fibre \& mineral'.
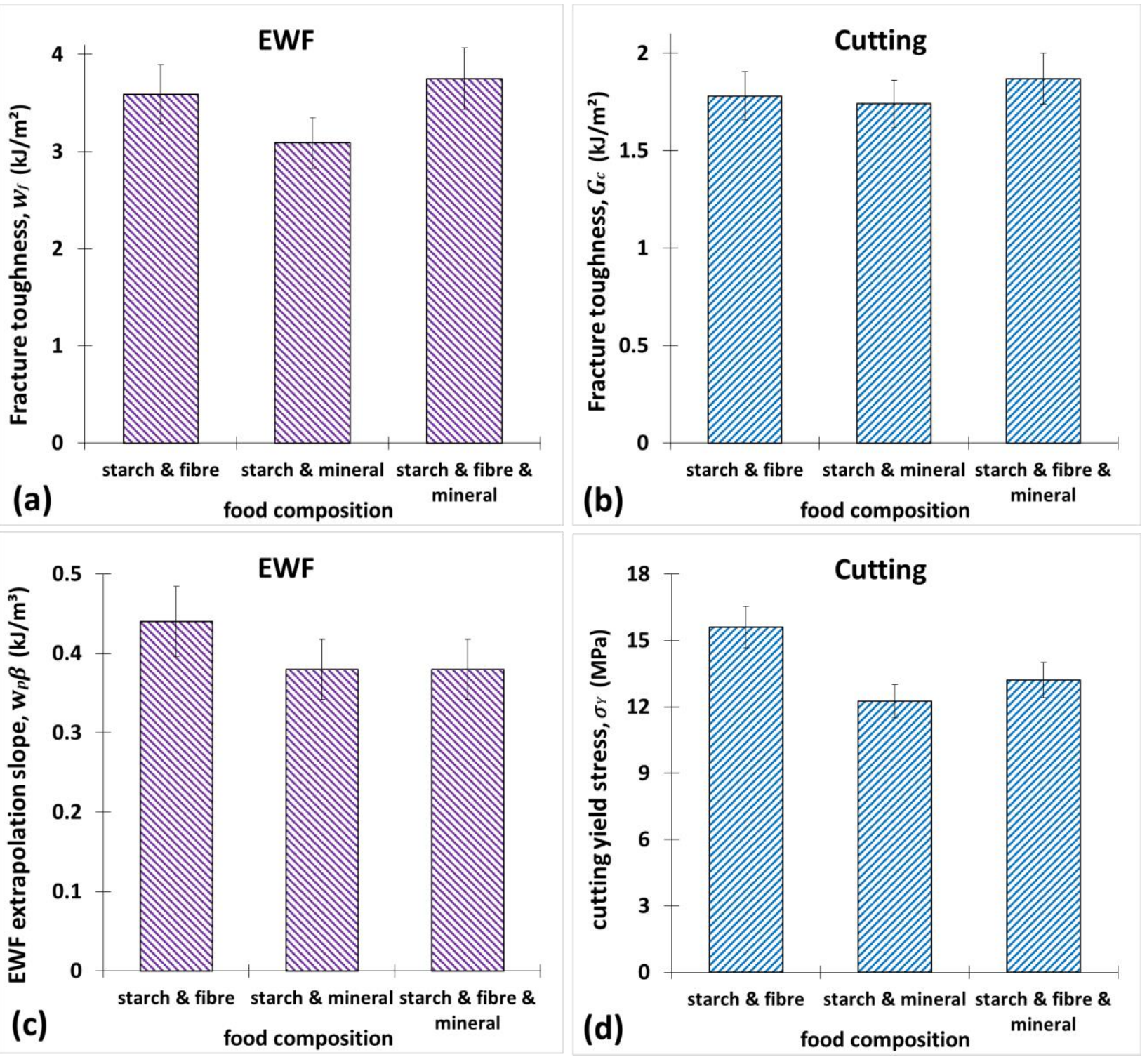

Figure 12. Comparison of the EWF against the cutting extrapolation results between three compositions: (a) EWF intercept (toughness), $w_{e}$, (b) cutting intercept (toughness), $G_{c}$, (c) EWF slope (specific inelastic work), $w_{p} \beta$, (d) cutting slope (yield stress), $\sigma_{Y}$. 


\subsection{Equivalence of the EWF and cutting tests}

The equivalence of the $w_{e}$ and $G_{c}$ terms derived from the EWF and cutting tests, respectively, has been a longstanding and challenging question. The first comparison of EWF-cutting data was only performed recently in (Skamniotis, Patel et al. 2016) with respect to the fundamental crack speed, $\dot{\alpha}$, variable, in a similar starch recipe. Two following differences were highlighted: firstly, $\dot{\alpha}$ is unlikely to be accurately controlled in EWF as it increases exponentially during crack propagation and furthermore depends on the applied crosshead speed, $\dot{\delta}$; in contrast, $\dot{\alpha}$ in cutting is directly controlled since the latter is equivalent to the applied cutting speed, $\dot{c}(\dot{\alpha}=\dot{c})$. Secondly, EWF gives $w_{e}$ as an average between crack initiation and propagation toughness compared to cutting which clearly deduces a $G_{c}$ toughness specific to crack propagation. However, by relating $\dot{\delta}$ with the corresponding average $\dot{\alpha}$ in EWF and plotting the $w_{e}$ and $G_{c}$ results as a function three $\dot{\alpha}$ values, a close agreement (9\% average $w_{e}-G_{c}$ difference) was found between the two tests, while despite the rate dependent nature this material, neither of the $w_{e}$ and $G_{c}$ were found dependent on $\dot{\alpha}$; the latter is also assumed in this study.

On the other hand, the above comparison was only performed for a single starch pet food recipe. Therefore, whether equivalency between EWF-cutting still holds for various compositions is assessed here; to allow for valid comparisons the data are treated as follows. Based on a recent study on similar starch recipes (Skamniotis, Kamaludin et al. 2017), the $w_{e}$ values derived via EWF here overestimate the true material fracture toughness and thus are less conservative than the cutting values (compare Figure 12(a) with 12(b)). This was expected mainly due to non-essential viscous energy dissipation embodied in Equation (4), inducing error both in the slope, $w_{p} \beta$, and intercept, $w_{e}$, of the EWF extrapolation. However, since the power law model fit indicated that the rate dependency in the stress-strain response is independent of composition (section 3.1 - Figure 3 ), it was reasonable to assume that the amount of viscous dissipation in the EWF specimens is also independent of composition. This implies that the error in the $w_{e}$ and $w_{p} \beta$ results is applied consistently between recipes and as a result comparison of the toughening effect measured via EWF and cutting over the compositions is assumed to be valid.

Based on the difference between the linear fit intercepts of the 'starch \& mineral' and 'starch $\&$ fibre \& mineral' recipes in Figure 12(b), the fibre toughening effect found in cutting is only $8 \%$, as opposed to the respective increase of $21 \%$ found in EWF (Figure 12(a)); this could be potentially caused by:

- anisotropy in fracture toughness,

- no fibre bridging in cutting.

Regarding anisotropy, while in the EWF tests the material is stretched along its extrusion (principal) axis, and thus the crack faces are perpendicular to that axis, the opposite occurs in cutting. Therefore, since here the fibre bridging effect is found to enhance toughness considerably, fibre orientation effects are expected to give an anisotropic toughness behaviour (Lü, Cheng et al. 2005)( $\mathrm{Li}$, Wang et al. 1991) and consequently different toughness results between EWF and cutting. Previous work (Skamniotis, Patel et al. 2016) reports on comparison between in-situ SEM tensile data on a similar starch recipe DENT samples stretched along the extrusion and the perpendicular axis. Although little difference was observed in the average force-displacement data, no fibre bridging was observed in the samples tested perpendicularly to the extrusion axis. The latter suggests a considerable degree of fibre orientation along the extrusion axis and thus potential anisotropy in fracture toughness. Due to a finite width of the sheet profile extrudates $(30 \mathrm{~mm})$ it 
was impossible to obtain specimens long enough such that the EWF method can be robustly performed perpendicularly to the extrusion axis. This would assist in characterising the anisotropic toughness behaviour and investigate further the comparability of the EWF and cutting methods in fibre reinforced soft polymers such the starch based food here.

Regarding the potential absence of fibre bridging effects in cutting, this is inherent to the cutting method. Specifically, in contrast to stiffer materials i.e. wood, the soft texture of starch gives rise to the blade-crack tip touching condition (Williams and Patel 2016), as shown in Figure 2(b). As a result, the moving blade may cut the fibres such that no bridging effects are allowed (Nairn , Skamniotis, Patel et al. 2016) providing misleading cutting results for toughness. Consequently the EWF method is rendered more appropriate for studying fibre toughening effects in soft matter.

\section{Conclusions}

This work investigates the significance of important food nutrients/constituents, namely minerals and fibres, with regards to the texture of starch extrudates based on tensile and fracture toughness data. Such studies are critical when both nutritional value and palatability need to be optimised in order to promote health in a range of pet breeds with diverse demands.

A comparison between four extruded pet food compositions is performed: pure starch, starch reinforced by cellulose fibres, starch reinforced by minerals, starch reinforced by both cellulose fibres and minerals. Overall, fibres and minerals are found to have a significant impact on the stiffness and toughness properties of starch even at low weight fractions of less than $5 \%$. The inclusion of fibres increases both stiffness due the stiff nature of cellulose and fracture toughness through the fibre bridging mechanism but decreases deformability in terms of tensile failure strain. In contrast, minerals alone show little effect on toughness and lead to a softer stress-strain response yet with larger failure strains. On the other hand, the Essential Work of Fracture (EWF) test showed a maximum $28 \%$ increase when fibres and minerals are added, attributed to a pronounced fibrematrix de-bonding toughening mechanism. Yet, if only fibres are included, a fibre breakage mechanism is also observed, which results into a less pronounced toughening effect.

Equivalency between the EWF and cutting tests, is found to hold only under certain circumstances. Here due to geometrical limitations of the extrudates, the two tests were applicable to measure toughness in different directions: cutting and EWF advanced the crack along the extrudate length and width, respectively. Thus, in anisotropic materials the two methods are not equivalent, while the EWF data appear more relevant to food oral breakdown. Secondly, cutting may impose an inevitable limitation in compliant matrices that contain fibres due to fibres being cut (fibre-cut) by the moving blade, not allowing fibre bridging toughening mechanisms to take place. Indeed, the fibre toughening effect here is only measured $8 \%$ in cutting, compared to a $21 \%$ in EWF. While this corresponds to a fibre content of $2.5 \% \mathrm{w} / \mathrm{w}$, cutting may significantly underestimate toughness at higher concentrations, not at least at larger fibre lengths. In such cases, EWF is superior over cutting and generally enables determining accurate toughness values in a 
wide range of materials. Instead, cutting is a more straightforward test, recommended for studying non-filled food matrices, especially when the slicing/cutting behaviour is of interest.

These findings provide guidelines and new options in food manufacturing i.e. calibrating texture not only via changing the extrusion parameters but also through altering composition. The experimental techniques employed are critical in designing extrusion-cooked foods where traditional fibre/particle reinforcement mechanisms are currently impossible to predict analytically or numerically, owing to: typical rate dependence and non-linearity of food matrices, fibre/particle irregular shape and random orientation. Such techniques may also help understand the link between the original mechanical-chemical-physical properties of products and the food oral and gastrointestinal breakdown rates.

\section{Acknowledgements}

The authors would like to acknowledge Mars Petcare for the financial support and for providing the samples as well as Mr. Ewan Armstrong who assisted in the cutting experiments. In addition the SEM in-situ micro-test device that was used in this work was purchased through funds from the Engineering and Physical Sciences Research Council (EP/C520629/1).

\section{References}

1. Ali, S. M., G. Unnikrishnan and M. Joseph (2013). "Essential work of fracture of low-filled poly (methyl methacrylate)/starch composites." Journal of Applied Polymer Science 128(3): 1409-1416.

2. Arenofsky, J. (2018). "Issue: The Pet Industry The Pet Industry."

3. Axelrod, H. R. (1993). Dog chew with modifiable texture, Google Patents.

4. Axelsson, E., A. Ratnakumar, M.-L. Arendt, K. Maqbool, M. T. Webster, M. Perloski, O. Liberg, J. M. Arnemo, Å. Hedhammar and K. Lindblad-Toh (2013). "The genomic signature of dog domestication reveals adaptation to a starch-rich diet." Nature 495(7441): 360-364.

5. Balaz, A., D. P. Bone and E. L. Shannon (1976). Method of making a dry fibrous meat-like pet food and composition thereof, Google Patents.

6. Barrangou, L. M., C. R. Daubert and E. A. Foegeding (2006). "Textural properties of agarose gels. I. Rheological and fracture properties." Food Hydrocolloids 20(2): 184-195.

7. Berthaume, M. A. (2016). "On the Relationship Between Tooth Shape and Masticatory Efficiency: A Finite Element Study." The Anatomical Record 299(5): 679-687.

8. Berthaume, M. A., E. R. Dumont, L. R. Godfrey and I. R. Grosse (2013). "How does tooth cusp radius of curvature affect brittle food item processing?" Journal of the Royal Society Interface 10(84): 20130240.

9. Bierer, T. L. (2007). "The Role of Biometrics in Canine Oral Health and Nutrition." Vet Med 1.

10. Blackman, B., T. Hoult, Y. Patel and J. Williams (2013). "Tool sharpness as a factor in machining tests to determine toughness." Engineering Fracture Mechanics 101: 47-58.

11. Bledzki, A. and J. Gassan (1999). "Composites reinforced with cellulose based fibres." Progress in polymer science 24(2): 221-274.

12. Bone, D. P. and E. L. Shannon (1975). Method of making a dry type pet food having a meatlike texture and composition thereof, Google Patents.

13. Bornhorst, G. M. and R. P. Singh (2012). "Bolus formation and disintegration during digestion of food carbohydrates." Comprehensive Reviews in Food Science and Food Safety 11(2): 101-118. 
14. Çakır, E., C. J. Vinyard, G. Essick, C. R. Daubert, M. Drake and E. A. Foegeding (2012). "Interrelations among physical characteristics, sensory perception and oral processing of protein-based soft-solid structures." Food Hydrocolloids 29(1): 234-245.

15. Chaléat, C. M., P. J. Halley and R. W. Truss (2008). "Properties of a plasticised starch blend. Part 1: Influence of moisture content on fracture properties." Carbohydrate Polymers 71(4): 535-543.

16. Chapman, H. and F. Pratt (1982). "Methods of analysis soils plant and water, university of California." Agriculture Division, USA 169.

17. Chen, J. (2009). "Food oral processing-A review." Food Hydrocolloids 23(1): 1-25.

18. Claes, J. E., L. De Maesschalck, V. Huysmans, H. Van Eyck, S. Schittecat, M. Pastuer and P. Moldenaers (2012). "Rheological study of breakfast replacing recipes used in the treatment of dysphagia." Journal of Texture Studies 43(2): 142-152.

19. Collyer, A. A. (2012). Rubber toughened engineering plastics, Springer Science \& Business Media.

20. COUNCII, N. R. (1985). Nutrient requirements of dogs, National Academies Press.

21. Forte, A., F. D'Amico, M. Charalambides, D. Dini and J. Williams (2015). "Modelling and experimental characterisation of the rate dependent fracture properties of gelatine gels." Food Hydrocolloids 46: 180-190.

22. Fu, S.-Y., X.-Q. Feng, B. Lauke and Y.-W. Mai (2008). "Effects of particle size, particle/matrix interface adhesion and particle loading on mechanical properties of particulate-polymer composites." Composites Part B: Engineering 39(6): 933-961.

23. Gamonpilas, C., M. Charalambides and J. Williams (2009). "Determination of large deformation and fracture behaviour of starch gels from conventional and wire cutting experiments." Journal of materials science 44(18): 4976-4986.

24. German, A. J., S. L. Holden, T. Bissot, P. J. Morris and V. Biourge (2010). "A high protein high fibre diet improves weight loss in obese dogs." The Veterinary Journal 183(3): 294-297.

25. Gierhart, D. L. and W. C. Hogan (1993). Flavor composition for pet food, Google Patents.

26. Goh, S., M. Charalambides and J. Williams (2005). "On the mechanics of wire cutting of cheese." Engineering fracture mechanics 72(6): 931-946.

27. Goh, S. M. (2002). An engineering approach to food texture studies, Imperial College London.

28. Hand, M. S., J. J. Hefferren, B. Marlow and L. D. Lewis (1995). Pet food product having oral care properties, Google Patents.

29. Holzapfel, G. A. and T. C. Gasser (2001). "A viscoelastic model for fiber-reinforced composites at finite strains: Continuum basis, computational aspects and applications." Computer methods in applied mechanics and engineering 190(34): 4379-4403.

30. Kong, F. and R. Singh (2008). "Disintegration of solid foods in human stomach." Journal of food science 73(5).

31. Lawrence, P. (1972). "Some theoretical considerations of fibre pull-out from an elastic matrix." Journal of Materials Science 7(1): 1-6.

32. Lepine, A. and G. A. Reinhart (1998). Pet food composition for large breed puppies and method for promoting proper skeletal growth, Google Patents.

33. Li, V. C., Y. Wang and S. Backer (1991). "A micromechanical model of tension-softening and bridging toughening of short random fiber reinforced brittle matrix composites." Journal of the Mechanics and Physics of Solids 39(5): 607-625.

34. Luke, D. and P. Lucas (1983). "The significance of cusps." Journal of oral rehabilitation 10(3): 197-206.

35. Marchal, Y., J.-F. Walhin and F. Delannay (1997). "Statistical procedure for improving the precision of the measurement of the essential work of fracture of thin sheets." International Journal of Fracture 87(2): 189-199. 
36. Mohammed P. Afandi, M., L. Wanigasooriya, S. Chakrabarti-Bell and M. Charalambides (2017). "Extrusion of unleavened bread dough: Experiments and simulations." Journal of Rheology 61(1): 49-65.

37. Mohammed P. Afandi, T., E, M. N. Charalambides and J. Williams (2013). "Mechanical characterization and micromechanical modeling of bread dough." Journal of Rheology (1978present) 57(1): 249-272.

38. Morren, S., T. Van Dyck, F. Mathijs, S. Luca, R. Cardinaels, P. Moldenaers, B. De Ketelaere and J. Claes (2015). "Applicability of the foodtexture puff device for rheological characterization of viscous food products." Journal of Texture Studies 46(2): 94-104.

39. Moscicki, L. (2011). Extrusion-cooking techniques: applications, theory and sustainability, John Wiley \& Sons.

40. Moscicki, L., M. Mitrus, A. Wojtowicz, T. Oniszczuk and A. Rejak (2013). Extrusion-Cooking of Starch.

41. Nahm Jr, L. J. (1982). Extruded fiber mixture pet food, Google Patents.

42. Nairn, J. A. "Numerical Modeling of Orthogonal Cutting: Application to Woodworking with a Bench Plane."

43. Norton, I., S. Moore and P. Fryer (2007). "Understanding food structuring and breakdown: engineering approaches to obesity." Obesity Reviews 8(s1): 83-88.

44. Nutrition, N. R. C. S. o. D. (1974). Nutrient requirements of dogs, National Academies.

45. Ockerman, H. W. and C. L. Hansen (1999). Animal by-product processing \& utilization, CRC Press.

46. Oksman, K., A. P. Mathew, R. Långström, B. Nyström and K. Joseph (2009). "The influence of fibre microstructure on fibre breakage and mechanical properties of natural fibre reinforced polypropylene." Composites Science and Technology 69(11): 1847-1853.

47. Patel, Y., B. Blackman and J. Williams (2009). "Determining fracture toughness from cutting tests on polymers." Engineering Fracture Mechanics 76(18): 2711-2730.

48. Patel, Y., B. Blackman and J. Williams (2009). "Measuring fracture toughness from machining tests." Proceedings of the Institution of Mechanical Engineers, Part C: Journal of Mechanical Engineering Science 223(12): 2861-2869.

49. Pera, P., C. Bucca, P. Borro, C. Bernocco, A. De Lillo and S. Carossa (2002). "Influence of mastication on gastric emptying." Journal of dental research 81(3): 179-181.

50. Pietrzyk, S., T. Fortuna, K. Królikowska, E. Rogozińska, M. Łabanowska and M. Kurdziel (2013). "Effect of mineral elements on physicochemical properties of oxidised starches and generation of free radicals." Carbohydrate polymers 97(2): 343-351.

51. Reinhart, G. A. (1997). Pet food product containing fermentable fibers and process for treating gastrointestinal disorders, Google Patents.

52. Saleemi, A. and J. Nairn (1990). "The plane-strain essential work of fracture as a measure of the fracture toughness of ductile polymers." Polymer Engineering \& Science 30(4): 211-218.

53. Schiessel, H., R. Metzler, A. Blumen and T. Nonnenmacher (1995). "Generalized viscoelastic models: their fractional equations with solutions." Journal of physics A: Mathematical and General 28(23): 6567.

54. Skamniotis, C., M. Elliott and M. Charalambides (2017). "On modeling the large strain fracture behaviour of soft viscous foods." Physics of Fluids 29(12): 121610.

55. Skamniotis, C., M. Kamaludin, M. Elliott and M. Charalambides (2017). "A novel essential work of fracture experimental methodology for highly dissipative materials." Polymer.

56. Skamniotis, C., Y. Patel, M. Charalambides and M. Elliott (2016). "Fracture investigation in starch-based foods." Interface focus 6(3): 20160005.

57. Sorrentino, A., G. Gorrasi and V. Vittoria (2007). "Potential perspectives of bionanocomposites for food packaging applications." Trends in Food Science \& Technology 18(2): 84-95. 
58. Szczesniak, A. (1990). "Psychorheolgoy and texture as factors controlling the consumer acceptance of food." Cereal Foods World 35(12): 1201-1205.

59. Taguet, A., M. N. Bureau, M. A. Huneault and B. D. Favis (2014). "Toughening mechanisms in interfacially modified HDPE/thermoplastic starch blends." Carbohydrate polymers 114: 222229.

60. Takaku, A. and R. Arridge (1973). "The effect of interfacial radial and shear stress on fibre pull-out in composite materials." Journal of Physics D: Applied Physics 6(17): 2038.

61. Tharakan, A., I. Norton, P. Fryer and S. Bakalis (2010). "Mass transfer and nutrient absorption in a simulated model of small intestine." Journal of food science 75(6).

62. Tran, Q. D., W. H. Hendriks and A. F. van der Poel (2008). "Effects of extrusion processing on nutrients in dry pet food." Journal of the Science of Food and Agriculture 88(9): 1487-1493.

63. Vandenberghe, E. and J. Claes (2011). "Sensory and instrumental analysis of the juiciness of strawberries." Journal of texture studies 42(1): 42-49.

64. Wan, Y., H. Luo, F. He, H. Liang, Y. Huang and X. Li (2009). "Mechanical, moisture absorption, and biodegradation behaviours of bacterial cellulose fibre-reinforced starch biocomposites." Composites Science and Technology 69(7-8): 1212-1217.

65. Wani, S. and P. Kumar (2016). "Effect of extrusion on the nutritional, antioxidant and microstructural characteristics of nutritionally enriched snacks." Journal of Food Processing and Preservation 40(2): 166-173.

66. Williams, J. and Y. Patel (2016). "Fundamentals of cutting." Interface focus 6(3): 20150108.

67. Williams, J. and M. Rink (2007). "The standardisation of the EWF test." Engineering fracture mechanics 74(7): 1009-1017.

68. Williams, J. G. (1984). "Fracture mechanics of polymers." Ellis Horwood Limited, Market Cross House, Cooper St, Chichester, West Sussex, PO 19, 1 EB, UK, 1984. 302.

69. Zicker, S. C. (2008). "Evaluating pet foods: how confident are you when you recommend a commercial pet food?" Topics in companion animal medicine 23(3): 121-126. 